NBS

PUBLICATIONS

$A \amalg I 105905818$

I A ANST OF STANDARDS \& TECH R.I.C.

NATL INST OF STANDARDS \& TECH R.|.

(I)

A11102902818
Bernal, Javiron constructing Delaunay
Bast- eau of Standards)

Bernal, Javier/On constructing 2198 C.1 NIST- eau of Standards)

NIST Technical Note 1252

On Constructing Delaunay Triangulations

for Sets Constrained by Line Segments

Javier Bernal

JF COMMERCE

tandards and Technology

eau of Standards) 



\section{NIST Technical Note 1252}

\section{On Constructing Delaunay Triangulations for Sets Constrained by Line Segments}

Javier Bernal

Mathematical Analysis Division

Center for Computing and Applied Mathematics

National Engineering Laboratory

National Institute of Standards and Technology

(formerly National Bureau of Standards)

Gaithersburg, MD 20899

\section{Issued September 1988}

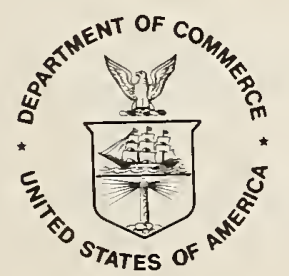

NOTE: As of 23 August 1988, the National Bureau of

Standards (NBS) became the National Institute of

Standards and Technology (NIST) when President

Reagan signed into law the Omnibus Trade and

Competitiveness Act.

U.S. Department of Commerce

C. William Verity, Secretary

National Institute of Standards and Technology

(formerly National Bureau of Standards)

Ernest Ambler, Director 
National Institute of Standards and Technology Technical Note 1252 33 pages (Sept. 1988) CODEN: NBTNAE
U.S. Government Printing Office Washington: 1988
For sale by the Superintendent of Documents U.S. Government Printing Office Washington, DC 20402 
CONTENTS

Page

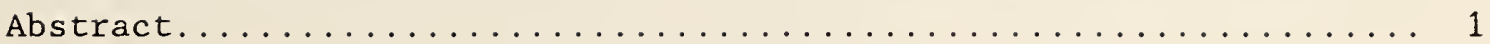

1. Introduction .............................. 2

2. The UPDTRI algorithm........................ 5

3. The EDGSTR algorithm....................... 10

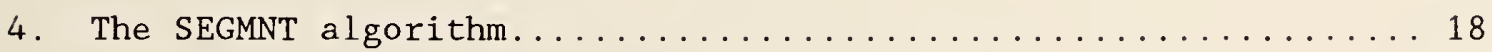

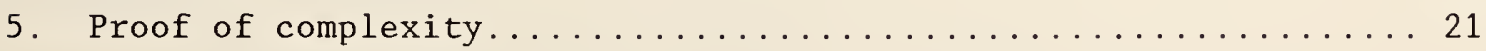

References................................... 29 



\title{
On constructing Delaunay triangulations for sets constrained by line segments
}

\author{
Javier Bernal \\ National Bureau of Standards, Gaithersburg, MD 20899, U. S. A.
}

\begin{abstract}
In this paper, we propose a simple algorithm for constructing a Delaunay triangulation for a finite set of points in the plane constrained by a finite collection of line segments. This algorithm constructs first a Delaunay triangulation for the set, and then generates from it a sequence of triangulations as each line segment is incorporated into the previously obtained triangulation. An expected time analysis shows the algorithm to be linear if the number of line segments is kept constant.

Key words: algorithm; computational complexity; computational geometry; constrained Delaunay triangulation; expected time analysis; multiply-connected polygonal regions; Voronoi diagram
\end{abstract}

AMS(MOS) subject classifications. 68U05 


\section{Introduction}

Let $S$ be a finite set of points in the plane. By a triangulation for $S$ we mean a finite collection of triangles in the plane having pair-wise disjoint interiors, each of which intersects $S$ exactly at its vertices, and the union of which is the convex hull of $S$. Given a triangulation $T$ for $S$, we say that $T$ is a Delaunay triangulation for $S$ if for each triangle in $T$ there does not exist a point of $S$ inside the circumcircle of the triangle. Delaunay triangulations have been thoroughly studied in $[8,11,12]$.

We say that a region in the plane is a multiply-connected polygonal region if it is a bounded and closed set whose interior is nonempty and connected, and whose boundary is the union of a finite number of closed line segments. Here and in the sequel, given a multiply-connected polygonal region $R$, we denote by $I N T(R), B N D(R)$, and $V T X(R)$, respectively, the interior of $R$, the boundary of $R$, and the set of vertices of the boundary of $R$. In addition, given points $P$ and $Q$ in the plane, $P \neq Q$, we denote by $[P, Q],(P, Q)$, $[P, Q)$, and $(P, Q]$, respectively, the closed, open, and half-open line segments that $P$ and $Q$ determine.

De Floriani, Falcidieno, and Pienovi [5] have studied the concept of a Delaunay triangulation for a finite set of points in the plane constrained by the boundary of a multiply-connected polygonal region. Let $R$ be a multiply-connected polygonal region, and let $S$ be a finite subset of $R$ that contains $V T X X(R)$. De Floriani, et al. define a triangulation for $S$ constrained by $B N D(R)$ as a finite collection of triangles having pair-wise disjoint interiors, each of which intersects $S$ exactly at its vertices, and the union of which is $R$. Given a triangulation $T$ for $S$ constrained by $B N D(R)$, De Floriani, et al. call $T$ a Delaunay triangulation for $S$ constrained by $B N D(R)$ if for each triangle in $T$ there does not exist a point of $S$ inside the circumcircle of the triangle such that the line segments that connect the point and the vertices of the triangle are contained in $R$.

Let $T$ be a triangulation for a set $S$ constrained by the boundary of a multiply-connected polygonal region $R$. Given a triangle $t$ in $T$, we denote by $A D J(t)$ the set of points in $S$ that are vertices of triangles in $T$ adjacent to $t$, and say that $t$ satisfies the circle criterion in $T$ if there does not exist a point in $A D J(t)$ inside the circumcircle of $t$. De Floriani, et al. state that using arguments similar to those developed in [6,8], it can be shown that $T$ is a Delaunay triangulation for $S$ constrained by $B N D(R)$ if each triangle in $T$ satisfies the circle criterion in $T$.

In this paper, we study a more general type of triangulation. Let $S$ be a finite set of points in the plane, and let $E$ be a finite collection, possibly empty, of line segments that intersect only at points in $S$ and whose endpoints belong to $S$. We say that a triangulation 
$T$ for $S$ is a triangulation for $S$ constrained by $E$ if for each $e$ in $E$ and each $t$ in $T$, $e$ does not intersect $I N T(t)$. Given $T$, a triangulation for $S$ constrained by $E$, we say that $T$ is a Delaunay triangulation for $S$ constrained by $E$ if for each $t$ in $T$ there does not exist a point $P$ of $S$ inside the circumcircle of $t$ such that no $e$ in $E$ intersects the interior of the convex hull of $t \cup\{P\}$.

Delaunay triangulations constrained by line segments have been studied by Lee [7] and Chew $[3,4]$ for line segments that do not cross. Lee and Chew have also shown how to construct them in $O\left(N(\log N)^{2}\right)$ and $O(N \log N)$ worst-case complexity, respectively, where $N$ is the number of points in $S$. In this paper, we present an algorithm, based on a different approach, that computes this type of triangulation in expected linear time for fixed $E$. It consists of two steps. In the first step, a Delaunay triangulation for $S$ is constructed by applying to $S$ one of several existing algorithms $[1,2,9,10]$. In the second step, a sequence of triangulations for $S$, the last of which is the desired triangulation, is generated from the initial triangulation as each line segment in $E$ is incorporated into the previously obtained triangulation.

Given a simple polygon $R$, and points $P_{0}$ and $P_{1}$ in $\operatorname{VTX}(R)$ such that $\left[P_{0}, P_{1}\right]$ is a linear component of $B N D(R)$, we say that $R$ is star-shaped relative to $\left[P_{0}, P_{1}\right]$ if for each point $P$ in $R \backslash\left[P_{0}, P_{1}\right]$, there exists a point $Q$ in $\left(P_{0}, P_{1}\right)$ such that $(P, Q)$ is contained in $I N T(R)$. We say that $R$ is edge star-shaped if it is star-shaped relative to at least one of the linear components of $B N D(R)$. Figure 1 illustrates an edge star-shaped simple polygon.

Given a region $R$ in the plane, we say that $R$ is a generalized multiply-connected polygonal region if it is a bounded and closed set whose interior is nonempty, and whose boundary is the union of a finite number of closed line segments. Given a generalized multiply-connected polygonal region $R$, for some unique positive integer $k$, let $R_{i}, i=1, \ldots, k$, be the unique multiply-connected polygonal regions that are the closures of the connected components of the interior of $R$. Let $S$ be a finite subset of $R$ that contains $\operatorname{VTX}\left(R_{i}\right)$ for each $i$, $i=1, \ldots, k$, and for each $i, i=1, \ldots, k$, let $S_{i}$ be the intersection of $S$ and $R_{i}$. Given for each $i, i=1, \ldots, k$, a triangulation $T_{i}$ for $S_{i}$ constrained by $B N D\left(R_{i}\right)$, we call the union of the triangulations $T_{i}, i=1, \ldots, k$, a triangulation for $S$ constrained by $B N D(R)$, where $B N D(R)$ denotes the boundary of $R$. We call it a Delaunay triangulation for $S$ constrained by $B N D(R)$, if for each $i, i=1, \ldots, k, T_{i}$ is a Delaunay triangulation for $S_{i}$ constrained by $B N D\left(R_{i}\right)$.

A crucial aspect of the algorithm we propose involves edge star-shaped simple polygons and generalized multiply-connected polygonal regions: the incorporation of each line segment of $E$ into the previously obtained triangulation for $S$. To do this, the algorithm first identifies those triangles in the triangulation whose interiors intersect the line segment. A 


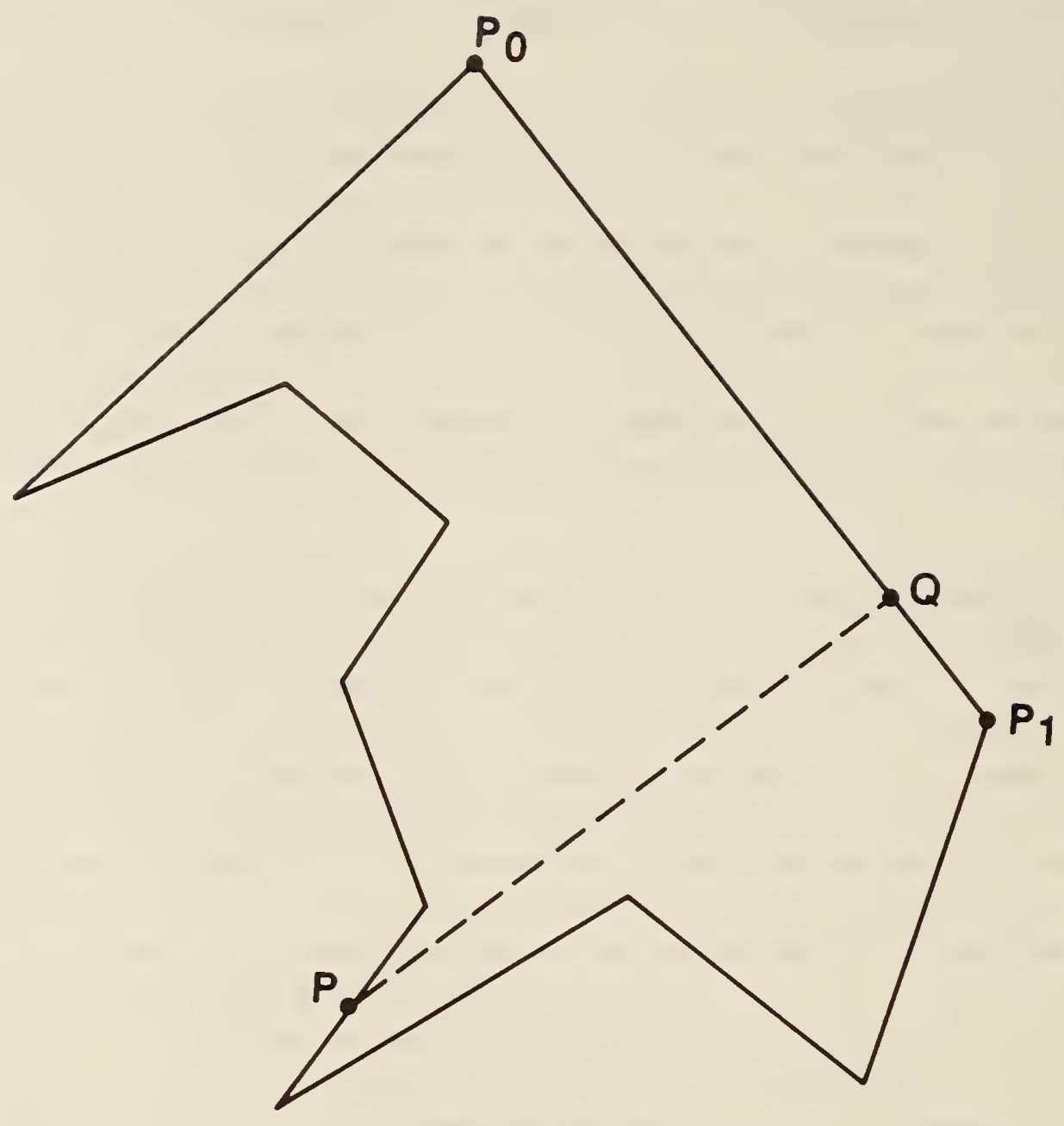

Figure 1: An edge star-shaped simple polygon. For each point $P$ in the complement of $\left[P_{0}, P_{1}\right]$ with respect to the polygon, there exists a point $Q$ in $\left(P_{0}, P_{1}\right)$ such that $(P, Q)$ is contained in the interior of the polygon. 
finite collection of edge star-shaped simple polygons with pair-wise disjoint interiors is then produced as the union of the identified triangles is intersected with each closed half-plane that the line segment determines. Next, for each polygon in this collection, a monotonically increasing (ordered by inclusion) finite sequence of generalized multiply-connected polygonal regions that converge to the polygon is produced; a Delaunay triangulation for the intersection of $S$ with each of these regions constrained by the boundary of the region is computed; and in the limit a Delaunay triangulation for the intersection of $S$ with the polygon constrained by the boundary of the polygon is obtained. Finally, a new triangulation for $S$ results as the triangulations obtained in the limit as above are appended to the previously obtained triangulation for $S$, and the triangles identified as having interiors that intersect the line segment in $E$ are eliminated from this triangulation.

Three algorithms, which we call SEGMNT, EDGSTR, and UPDTRI, are outlined and justified in this paper. Algorithm SEGMNT, which makes use of algorithm EDGSTR, constructs a Delaunay triangulation for a finite set of points in the plane constrained by a finite collection of line segments. Algorithm EDGSTR, which makes use of algorithm UPDTRI, computes a Delaunay triangulation for a specialized subset of the boundary of an edge star-shaped simple polygon constrained by the boundary of the polygon. Finally, algorithm UPDTRI constructs a Delaunay triangulation for a set constrained by the boundary of a generalized multiply-connected polygonal region from a specialized triangulation for the set constrained by the same boundary. In Sections 2, 3, and 4, we outline and justify algorithms UPDTRI, EDGSTR, and SEGMNT, respectively. In Section 5, we show SEGMNT to be of expected linear complexity if the cardinality of the collection of line segments is kept constant. Finally, in Section 6, we offer a summary of the results presented in this paper.

\section{The UPDTRI algorithm}

Consider a generalized multiply-connected polygonal region $R$, and for some positive integer $k$, let $R_{i}, i=1, \ldots, k$, be the multiply-connected polygonal regions that are the closures of the connected components of the interior of $R$. Let $S$ be a finite subset of $R$ that contains $V T X\left(R_{i}\right)$ for each $i, i=1, \ldots, k$. For each $i, i=1, \ldots, k$, let $S_{i}$ be the intersection of $S$ and $R_{i}$, and let $T_{i}$ be a triangulation for $S_{i}$ constrained by $B N D\left(R_{i}\right)$ such that for an integer $l$, $1 \leq l \leq k$, a triangle $t^{*}$ in $T_{l}$, and a vertex $P^{*}$ of $t^{*}$, the following conditions hold:

1. If for some triangle $t^{\prime}$ in $T_{l}, t^{\prime} \neq t^{*}, P^{*}$ is inside the circumcircle of $t^{\prime}$, and the line segments that connect $P^{*}$ and the vertices of $t^{\prime}$ are contained in $R_{l}$, then one of these line segments intersects $I N T\left(t^{*}\right)$. 
2. For each triangle $t^{\prime}$ in $T_{l}, t^{\prime} \neq t^{*}$, there does not exist a point of $S_{l}$ different from $P^{*}$ inside the circumcircle of $t^{\prime}$ such that the line segments that connect the point and the vertices of $t^{\prime}$ are contained in $R_{l}$.

3. For each $i, 1 \leq i \leq k, i \neq l, T_{i}$ is a Delaunay triangulation for $S_{i}$ constrained by $B N D\left(R_{i}\right)$.

With $T$ defined as the union of the triangulations $T_{i}, i=1, \ldots, k$, we present and justify algorithm UPDTRI, which produces from $T, t^{*}$, and $P^{*}$, a Delaunay triangulation $T^{*}$ for $S$ constrained by $B N D(R)$. Here and in the sequel, given a triangle $t$ with vertices $P_{1}, P_{2}, P_{3}$, in the order in which they appear in $B N D(t)$ in a counterclockwise direction around $I N T(t)$, we denote $t$ by $\triangle P_{1} P_{2} P_{3}$ and say that $\triangle P_{1} P_{2} P_{3}$ identifies $t$. Based on this convention, we let $P^{\prime}$ and $P^{\prime \prime}$ be points in $S_{l}$ for which $\Delta P^{*} P^{\prime} P^{\prime \prime}$ identifies $t^{*}$. The outline of UPDTRI follows.

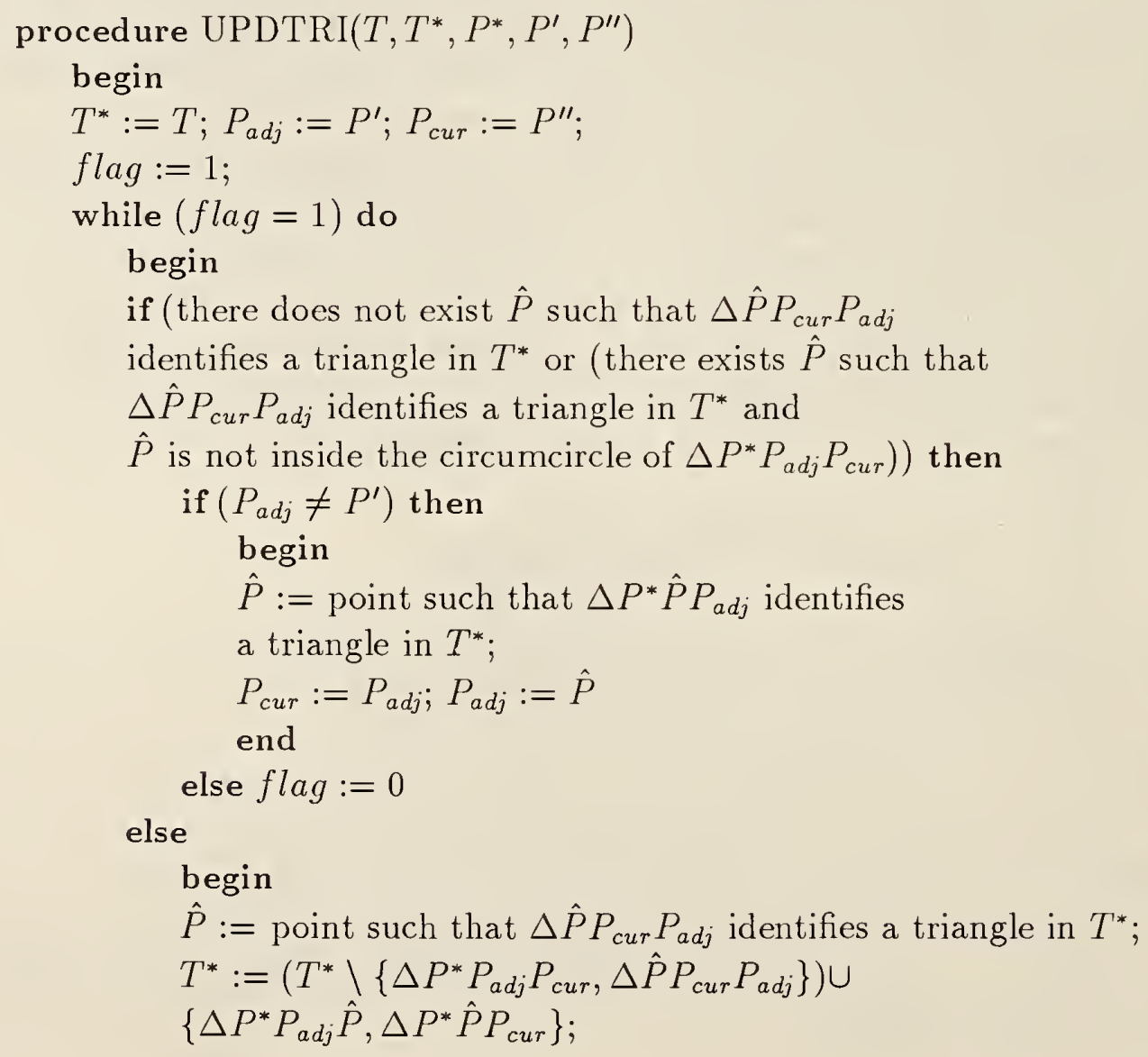




$$
\begin{aligned}
& \qquad \begin{array}{l}
P_{a d j}:=\hat{P} \\
\text { end }
\end{array} \\
& \text { end } \\
& \text { end }
\end{aligned}
$$

Justification of UPDTRI. That the execution of UPDTRI produces a triangulation $T^{*}$ from $T$ follows from the outline of the algorithm. Thus, assume that UPDTRI has been executed. For each $i, i=1, \ldots, k$, let $T_{i}^{*}$ be the set of triangles in $T^{*}$ whose vertices are all in $S_{i}$. Since $R$ is a generalized multiply-connected polygonal region, it follows from the outline of UPDTRI that for each $i, i=1, \ldots, k, i \neq l, T_{i}^{*}$ equals $T_{i}$, so that by the third condition stated above, each triangle in $T_{i}^{*}$ satisfies the circle criterion in $T_{i}^{*}$. Also, from the outline of UPDTRI, by the first and second condition stated above, each triangle in $T_{l}^{*} \cap T_{l}$ satisfies the circle criterion in $T_{l}^{*}$. Thus, if $T_{l}^{*} \neq T_{l}$, it suffices to show that each triangle in $T_{l}^{*} \backslash T_{l}$ satisfies the circle criterion in $T_{l}^{*}$. We assume this is not the case. Therefore, since $P^{*}$ is a vertex of every triangle in $T_{l}^{*} \backslash T_{l}$, points $Q^{\prime}, Q^{\prime \prime}$, and $Q^{\prime \prime \prime}$ exist in $S_{l}$ such that $\Delta P^{*} Q^{\prime} Q^{\prime \prime}$ and $\triangle P^{*} Q^{\prime \prime} Q^{\prime \prime \prime}$ identify triangles in $T_{l}^{*} \backslash T_{l}$ with $Q^{\prime \prime \prime}$ inside the circumcircle of $\Delta P^{*} Q^{\prime} Q^{\prime \prime}$. Clearly, it follows that a triangle can be identified with $\Delta Q^{\prime} Q^{\prime \prime} Q^{\prime \prime \prime}$ and $P^{*}$ is not inside the circumcircle of this triangle.

Let $\hat{Q}^{\prime}$ and $\hat{Q}^{\prime \prime \prime}$ be points in $S_{l}$ such that $\Delta \hat{Q}^{\prime} Q^{\prime \prime} \hat{Q}^{\prime \prime \prime}$ identifies the first triangle in $T_{l}$ eliminated by the algorithm with $Q^{\prime \prime}$ as a vertex.

If $\hat{Q}^{\prime}$ equals $Q^{\prime}$ and $\hat{Q}^{\prime \prime \prime}$ equals $Q^{\prime \prime \prime}$ then $P^{*}$ is not inside the circumcircle of $\Delta \hat{Q}^{\prime} Q^{\prime \prime} \hat{Q}^{\prime \prime \prime}$, a contradiction to the definition of $\Delta \hat{Q}^{\prime} Q^{\prime \prime} \hat{Q}^{\prime \prime \prime}$.

If $\hat{Q}^{\prime}$ does not equal $Q^{\prime}$ then from the outline of UPDTRI and the definitions of $Q^{\prime}$ and $Q^{\prime \prime}$, there exists a point $Q^{*}$ in $S_{l}$ separated from $Q^{\prime \prime}$ by the line through $P^{*}$ and $Q^{\prime}$ such that $\triangle Q^{*} Q^{\prime} Q^{\prime \prime}$ identifies the first triangle in $T_{l}$ eliminated by the algorithm with $Q^{\prime}$ as a vertex.

Figure 2 illustrates an example of this situation.

Clearly, $\left[Q^{\prime}, Q^{\prime \prime \prime}\right]$ and $\left[Q^{\prime \prime}, Q^{\prime \prime \prime}\right]$ are contained in $R_{l}$, and we show that so is $\left[Q^{*}, Q^{\prime \prime \prime}\right]$. Let $C_{1}$ and $C_{2}$ be the quadrilaterals with vertices $P^{*}, Q^{\prime}, Q^{\prime \prime}, Q^{\prime \prime \prime}$, and $P^{*}, Q^{*}, Q^{\prime}, Q^{\prime \prime}$, respectively. Since the line through $P^{\prime}$ and $P^{\prime \prime}$ separates $P^{*}$ from $Q^{\prime}, Q^{\prime \prime}, Q^{\prime \prime \prime}$, and $Q^{*}$, and $C_{1}$ and $C_{2}$ are convex, it follows that $C_{1} \cup C_{2}$ is convex so that $\left[Q^{*}, Q^{\prime \prime \prime}\right]$ is contained in $C_{1} \cup C_{2}$. Thus, $\left[Q^{*}, Q^{\prime \prime \prime}\right]$ is contained in $R_{l}$, and by the second condition stated above $Q^{\prime \prime \prime}$ is not inside the circumcircle of $\Delta Q^{*} Q^{\prime} Q^{\prime \prime}$. In addition, since triangles can be identified with $\triangle Q^{*} Q^{\prime} Q^{\prime \prime}, \Delta Q^{\prime} Q^{\prime \prime} Q^{\prime \prime \prime}$, and $\Delta P^{*} Q^{\prime} Q^{\prime \prime}, Q^{*}$ and $Q^{\prime \prime \prime}$ lie in the half-plane that contains $P^{*}$ and that is determined by the line through $Q^{\prime}$ and $Q^{\prime \prime}$. Therefore, as shown by the example in Figure 3 , the circumcircle of $\Delta Q^{\prime} Q^{\prime \prime} Q^{\prime \prime \prime}$ contains the part of the circumcircle of $\Delta Q^{*} Q^{\prime} Q^{\prime \prime}$ that is contained in this half-plane. Thus, by the definition of $\triangle Q^{*} Q^{\prime} Q^{\prime \prime}, P^{*}$ is inside the 


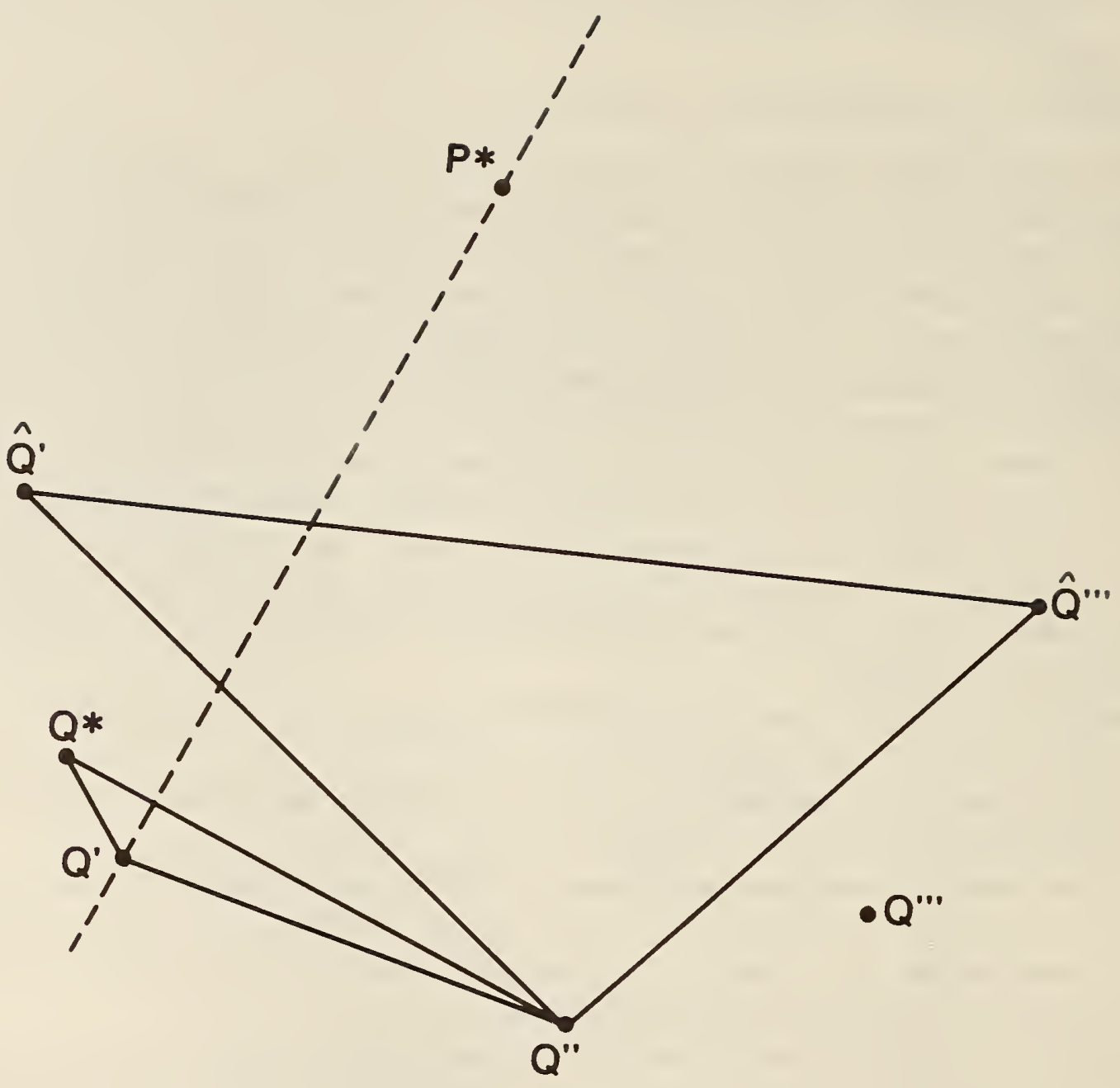

Figure 2: $\Delta \hat{Q}^{\prime} Q^{\prime \prime} \hat{Q}^{\prime \prime \prime}$ and $\Delta Q^{*} Q^{\prime} Q^{\prime \prime}$ when $\hat{Q}^{\prime}$ does not equal $Q^{\prime}$. The line through $P^{*}$ and $Q^{\prime}$ separates $Q^{*}$ and $Q^{\prime \prime}$. 


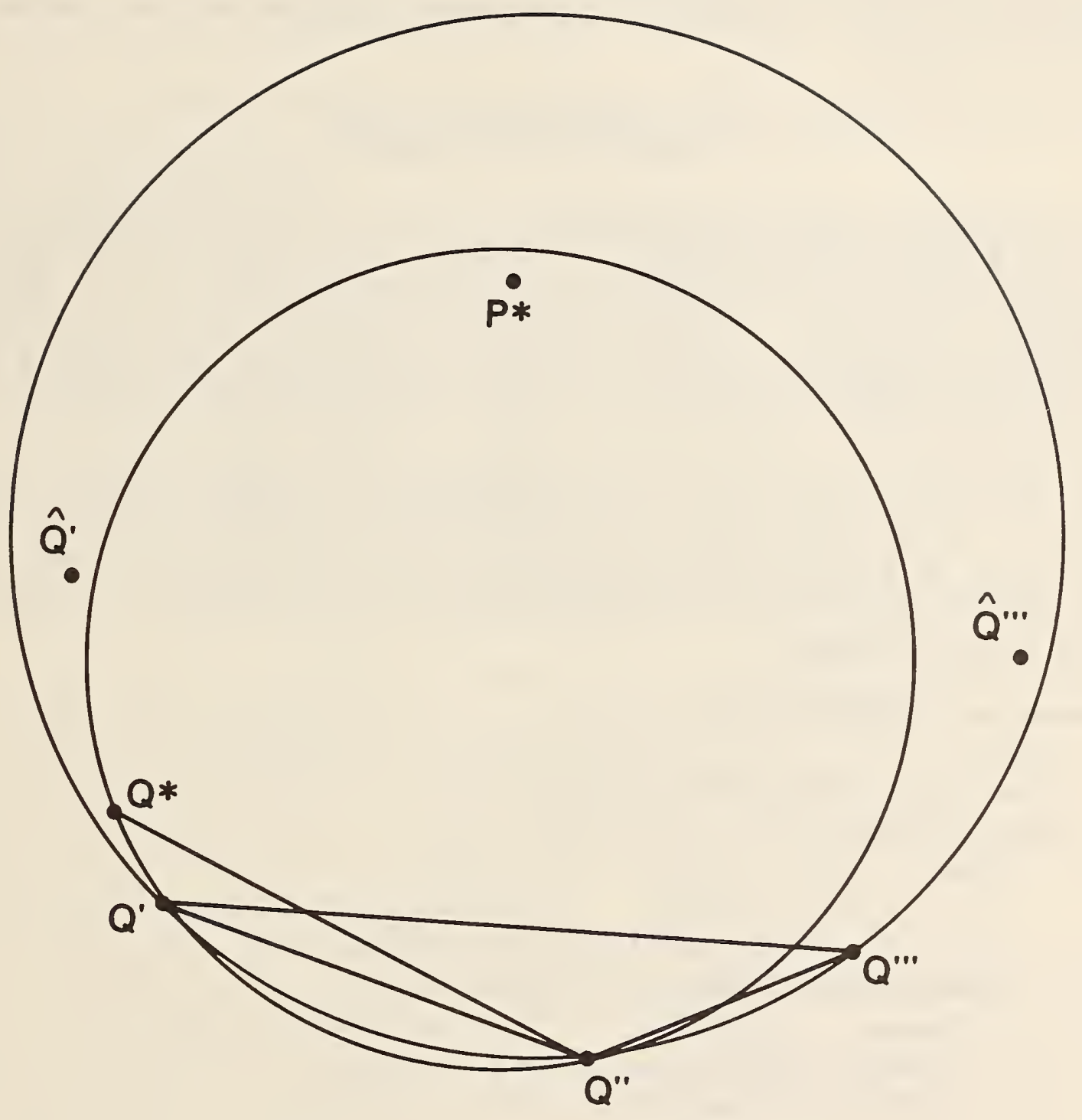

Figure 3: The circumcircles of $\triangle Q^{\prime} Q^{\prime \prime} Q^{\prime \prime \prime}$ and $\triangle Q^{*} Q^{\prime} Q^{\prime \prime}$. 
circumcircle of $\Delta Q^{\prime} Q^{\prime \prime} Q^{\prime \prime \prime}$, a contradiction.

Similarly, if $\hat{Q}^{\prime \prime \prime}$ does not equal $Q^{\prime \prime \prime}$, a contradiction is obtained, and the justification of UPDTRI is complete.

\section{The EDGSTR algorithm}

Let $R$ be an edge star-shaped simple polygon, and let $e$ be a linear component of $B N D(R)$ such that $R$ is star-shaped relative to $e$. Let $S$ be a finite subset of $B N D(R)$ such that $S$ contains $\operatorname{VTX}(R)$ and does not intersect the relative interior of $e$. Let $r$ be the number of points in $S$, and let $P_{1}, \ldots, P_{r}$ be the points in $S$ in the order in which they appear in $B N D(R)$ in a counterclockwise direction around $I N T(R)$ with $\left[P_{1}, P_{r}\right]$ equal to $e$. Define a one-to-one function $F$ from $\{1, \ldots, r\}$ onto $S$ by setting $F(i)$ equal to $P_{i}$ for each $i$, $i=1, \ldots, r$.

In what follows, we present and justify algorithm EDGSTR which computes a Delaunay triangulation $T$ for $S$ constrained by $B N D(R)$. Here, given points $Q_{1}, Q_{2}, Q_{3}$ in the plane, $Q_{2} \neq Q_{3}$ and $Q_{2} \neq Q_{1}$, we let $m\left(Q_{2} \vec{Q}_{3}, Q_{2} \vec{Q}_{1}\right)$ represent the size in radians of the angle produced by a counterclockwise rotation around $Q_{2}$ from ray $Q_{2} \vec{Q}_{3}$ to ray $Q_{2} \vec{Q}_{1}$. The outline of EDGSTR follows.

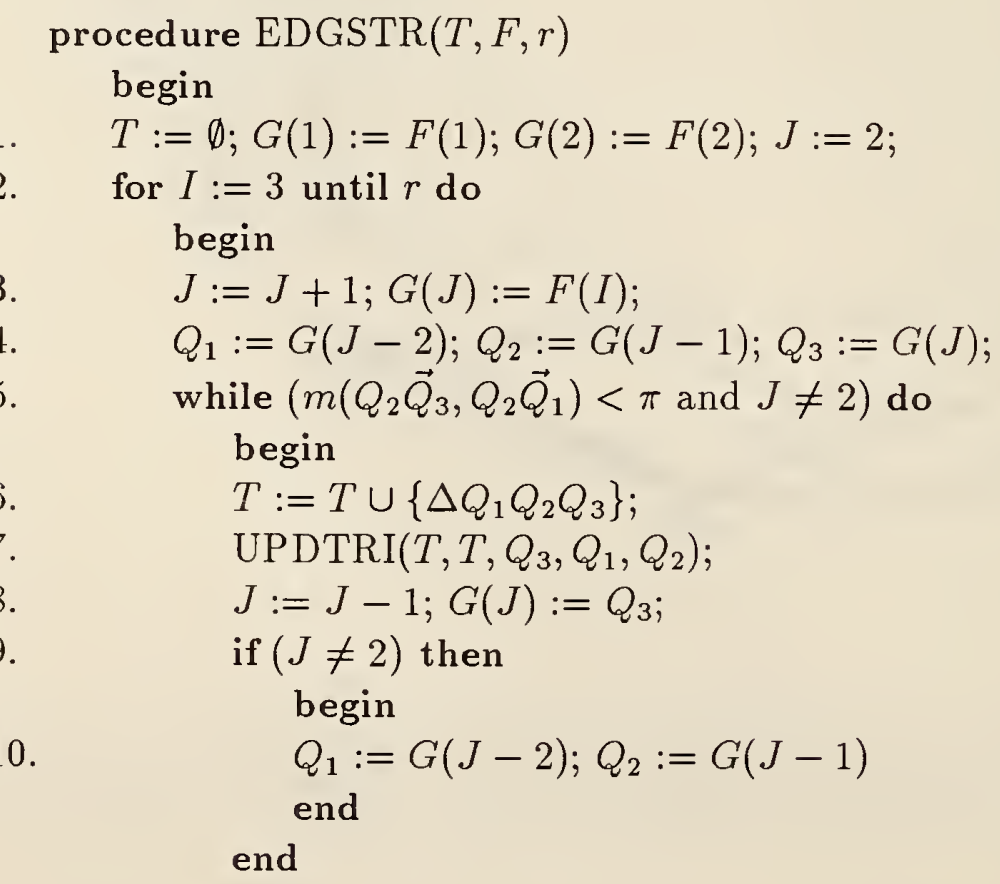




\section{end \\ end}

Justification of EDGSTR. We show that the execution of EDGSTR produces a collection of triangles $T$ that is a Delaunay triangulation for $S$ constrained by $B N D(R)$. First, we must prove the following observation:

Given integers $j, k, l, 1 \leq j<k<l \leq r$, if $R$ contains $\left[P_{j}, P_{k}\right]$ and $\left[P_{k}, P_{l}\right], m\left(P_{k} \vec{P}_{l}, P_{k} \vec{P}_{j}\right)$ is less than $\pi$, and $\left[P_{j}, P_{k}\right]$ and $\left[P_{k}, P_{l}\right]$ intersect $S$ exactly at $P_{j}$ and $P_{k}$, and $P_{k}$ and $P_{l}$, respectively, then $\triangle P_{j} P_{k} P_{l}$ identifies a triangle that is contained in $R$ and that intersects $S$ exactly at $P_{j}, P_{k}$, and $P_{l}$. In addition, given a finite collection of triangles $T$ with pair-wise disjoint interiors, each of which is contained in $R$ and intersects $S$ exactly at its vertices, if $\left(P_{j}, P_{k}\right)$ and $\left(P_{k}, P_{l}\right)$ do not intersect the interior of each triangle in $T$, and $I N T\left(\Delta P_{j} P_{k} P_{l}\right)$ does not intersect the interior of each triangle in $T$ with $P_{k}$ as a vertex, then $I N T\left(\Delta P_{j} P_{k} P_{l}\right)$ does not intersect the interior of any triangle in $T$.

Figure 4 illustrates an example of $R, P_{j}, P_{k}, P_{l}, P_{1}, P_{r}$, as described above.

In what follows, we prove the first part of the observation. The second part will follow from it.

That $\triangle P_{j} P_{k} P_{l}$ identifies a triangle follows from the fact that $m\left(P_{k} \vec{P}_{l}, P_{k} \vec{P}_{j}\right)$ is less than $\pi$. It follows from the ordering of $P_{i}, i=1, \ldots, r$, in $B N D(R)$, that in order to prove the rest of the first part of the observation, it suffices to show that for each integer $i, 1 \leq i \leq j$ or $l \leq i \leq r, P_{i}$ does not belong to $C \equiv \Delta P_{j} P_{k} P_{l} \backslash\left(\left[P_{j}, P_{k}\right] \cup\left[P_{k}, P_{l}\right]\right)$. We assume the contrary and let $H$ be the set of integers $i$ at which $m\left(P_{l} \vec{P}_{i}, P_{l} \vec{P}_{k}\right)$ attains its minimum value, with $1 \leq i \leq j$ or $l \leq i \leq r$, and $P_{i}$ in $C$. Clearly, each $i$ in $H$ must be different from $j$ and $l$, so that either $1 \leq i<j$ or $l<i \leq r$ for each $i$ in $H$.

If $l<i \leq r$ for some $i$ in $H$, we show that for each point $Q$ in $\left(P_{1}, P_{r}\right),\left(P_{l}, Q\right)$ is not entirely contained in $I N T(R)$, a contradiction to the fact that $R$ is star-shaped relative to $\left[P_{1}, P_{r}\right]$. Thus, let $i^{*}$ be an integer in $H$ such that $l<i^{*} \leq r$. It follows that the ray $P_{l} \vec{P}_{i^{*}}$ intersects $\left[P_{j}, P_{k}\right)$. Thus, there exists an integer $n, j \leq n<k$, and a point $P^{*}$ in $B N D(R)$, such that $P_{l} \vec{P}_{i^{*}}$ intersects $\left[P_{n}, P_{n+1}\right)$ exactly at $P^{*}$, and $\left[P^{*}, P_{i^{*}}\right]$ is contained in $R$. Let $R^{\prime}$ be the simple polygon whose vertices are contained in $\left\{P^{*}\right\} \cup\left\{P_{i}, n<i \leq i^{*}\right\}$ and whose boundary contains $P^{*}, P_{i}, i=n+1, \ldots, i^{*}$, in this order in a counterclockwise direction around its interior. Figure 5 illustrates an example of $R^{\prime}, P_{j}, P_{k}, P_{l}, P_{i^{*}}, P_{n}, P_{n+1}, P^{*}, P_{1}, P_{r}$. Let $Q$ be a point in $\left(P_{1}, P_{r}\right)$ and assume that $\left(P_{l}, Q\right)$ is contained in $I N T(R)$. It follows from the definition of $R^{\prime}$ that $\left[P_{l}, Q\right]$ must intersect $I N T\left(R^{\prime}\right)$. However, from the definitions of $i^{*}, P^{*}$, and the ordering of $P_{i}, i=1, \ldots, r$, in $\operatorname{BND}(\mathrm{R}), Q$ is not contained in $I N T\left(R^{\prime}\right)$, so that $\left[P_{l}, Q\right]$ must intersect $B N D\left(R^{\prime}\right)$ at a point other than $P_{l}$. Every point in $B N D\left(R^{\prime}\right)$ 


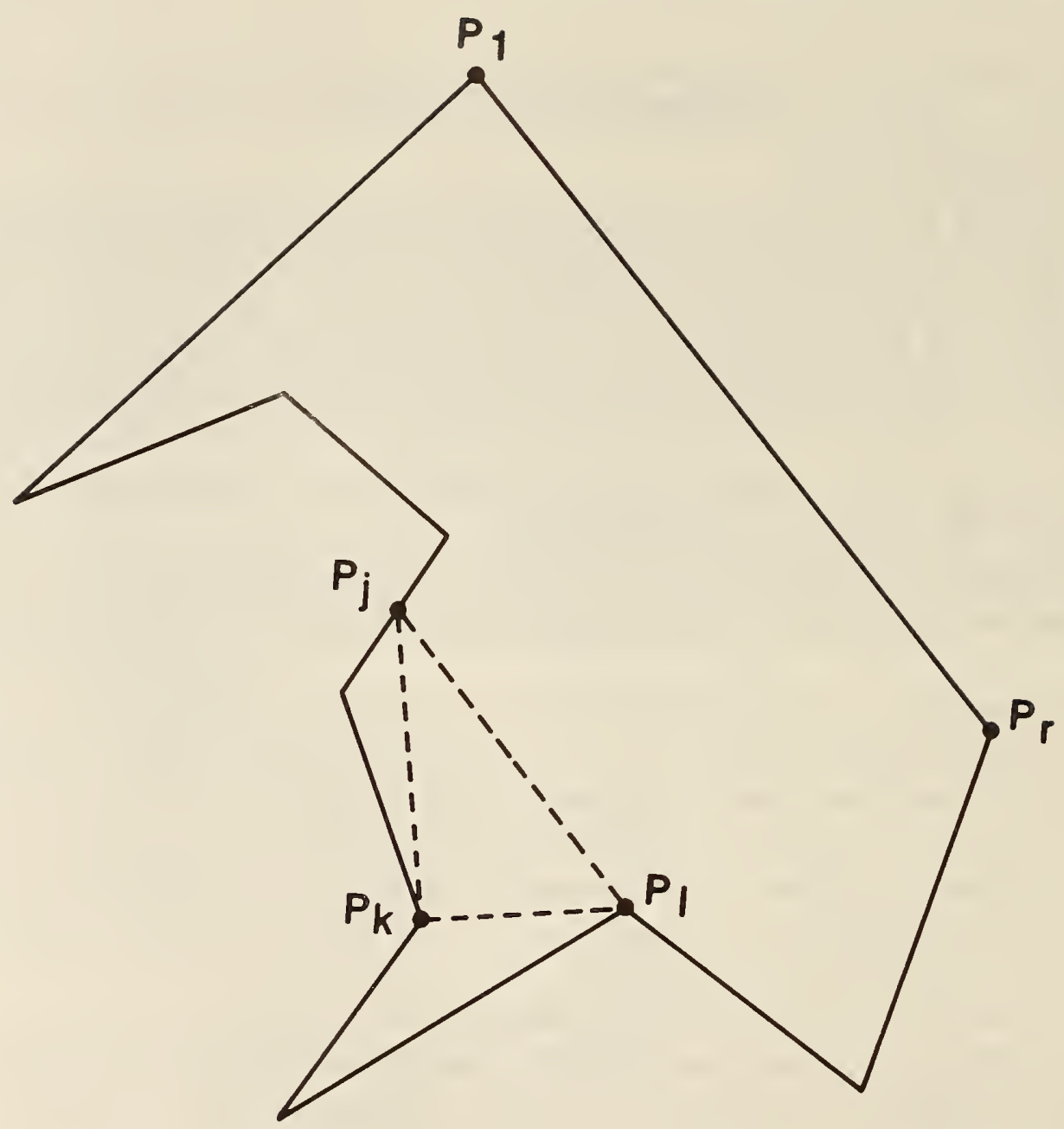

Figure 4: The polygon $R$ and the points $P_{j}, P_{k}, P_{l}, P_{1}, P_{r} . \quad \Delta P_{j} P_{k} P_{l} \subseteq R$ and $\triangle P_{j} P_{k} P_{l} \cap S=\left\{P_{j}, P_{k}, P_{l}\right\}$. 


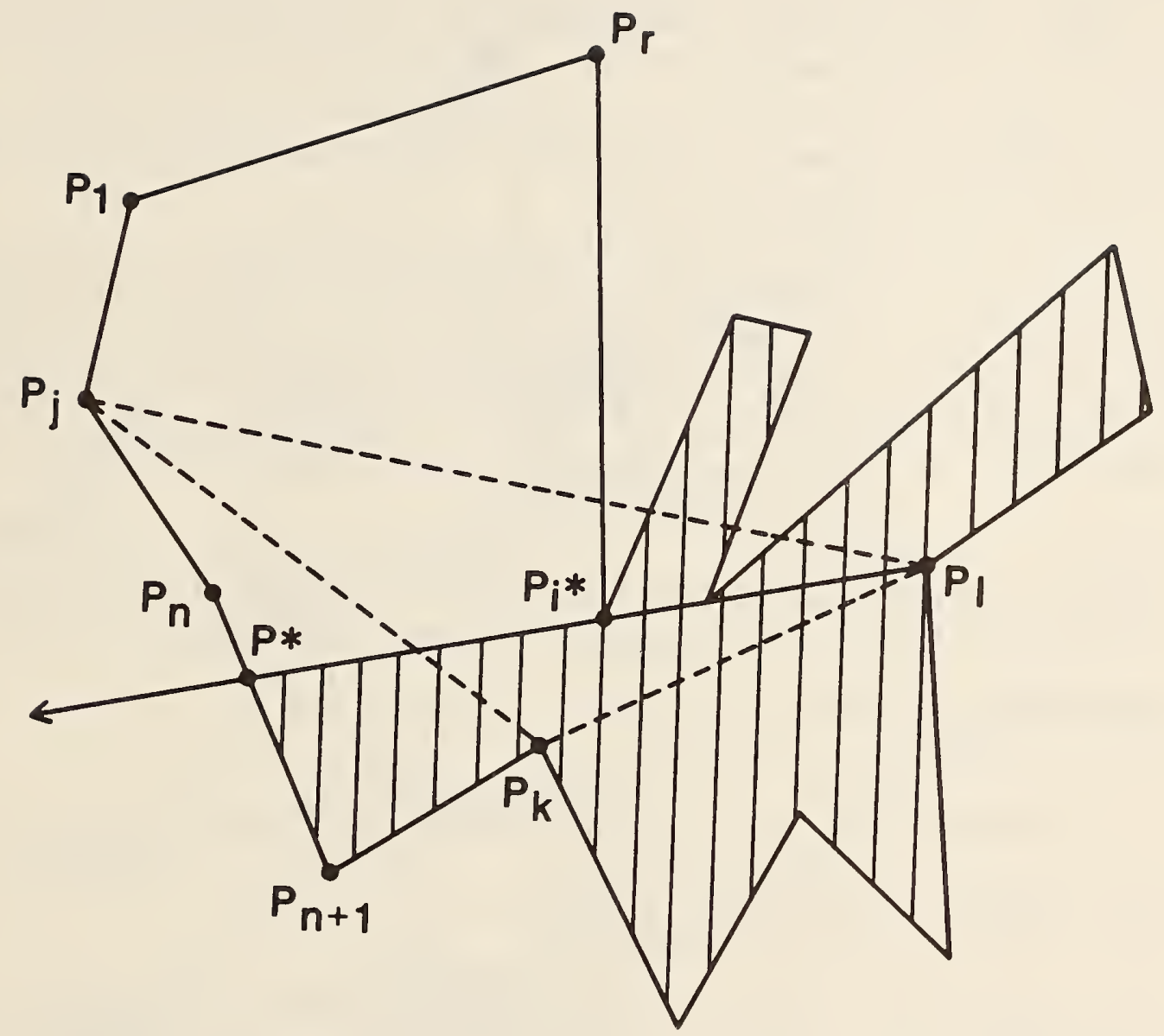

Figure 5: The polygon $R^{\prime}$ (shaded region) and the points $P_{j}, P_{k}, P_{l}, P_{i^{*}}, P_{n}, P_{n+1}, P^{*}, P_{1}$, $P_{r}$. The ray $P_{l} \vec{P}_{i^{*}}$ intersects $\left[P_{n}, P_{n+1}\right)$ at $P^{*}$. 
that is not in $\left(P^{*}, P_{i^{*}}\right)$ belongs to $B N D(R)$ and differs from $Q$. Thus, $\left[P_{l}, Q\right]$ must intersect $\left(P^{*}, P_{i^{*}}\right)$. But $P^{*}, P_{i^{*}}$, and $P_{l}$ are colinear with $P_{i^{*}}$ in $\left(P^{*}, P_{l}\right)$, so that $\left(P_{l}, Q\right)$ must contain $P_{i^{*}}$. Since $Q$ is an arbitrary point in $\left(P_{1}, P_{r}\right)$, we have the desired contradiction.

Similarly, if $1 \leq i<j$ for each $i$ in $H$, it can be shown that for each point $Q$ in $\left(P_{1}, P_{r}\right)$, $\left(P_{j}, Q\right)$ is not entirely contained in $\operatorname{INT}(R)$. This is also a contradiction, and it completes the proof of the first part of the observation.

Let $I, J, G$, and $T$ be as defined in the outline of EDGSTR. Let $K$ be a variable which is set to zero at the start of the execution of EDGSTR and which is incremented by one at the start of each execution of line 6 of EDGSTR. It follows from the outlines of EDGSTR and UPDTRI, the above observation, the definition of $R$, and an induction process on $K$, that at the start of each execution of line 6 , the criteria listed below are met. Figure 6 illustrates an example of $R, F, G, T, I, J$ that satisfies these criteria. The criteria follow:

1. $\{G(j), j=1, \ldots, J\} \subseteq\{F(i), i=1, \ldots, I\}, G(1)$ and $G(J)$ equal $F(1)$ and $F(I)$, respectively, and given integers $k, l, k^{\prime}, l^{\prime}, 1 \leq k, l \leq I, 1 \leq k^{\prime}, l^{\prime} \leq J$, such that $F(k)$ and $F(l)$ equal $G\left(k^{\prime}\right)$ and $G\left(l^{\prime}\right)$, respectively, then $k<l$ if and only if $k^{\prime}<l^{\prime}$.

2. If $J>3$ then $m(G(j) \vec{G}(j+1), G(j) \vec{G}(j-1)) \geq \pi$ for each $j$, $j=2, \ldots, J-2$.

3. The triangles in $T$ have pair-wise disjoint interiors.

4. Each triangle in $T$ is contained in $R$ and intersects $\{F(i), i=1, \ldots, I\}$ exactly at its vertices.

5. Given integers $k, l, 1 \leq k<l \leq I$ such that $[F(k), F(l)]$ is an edge of a triangle in $T$, if $l$ equals $k+1$ or if for some integer $j, 1 \leq j \leq J-1, F(k)$ and $F(l)$ equal $G(j)$ and $G(j+1)$, respectively, then $[F(k), F(l)]$ is an edge of exactly one triangle in $T$. Otherwise, it is an edge of two triangles in $T$.

6. Given an integer $i, 1 \leq i \leq I-1,[F(i), F(i+1)]$ is not an edge of a triangle in $T$ if and only if for some integer $j, 1 \leq j \leq J-1, F(i)$ and $F(i+1)$ equal $G(j)$ and $G(j+1)$, respectively.

7. Given an integer $j, 1 \leq j \leq J-1,[G(j), G(j+1)]$ is not an edge of a triangle in $T$ if and only if for some integer $i, 1 \leq i \leq I-1, G(j)$ and $G(j+1)$ equal $F(i)$ and $F(i+1)$, respectively. 


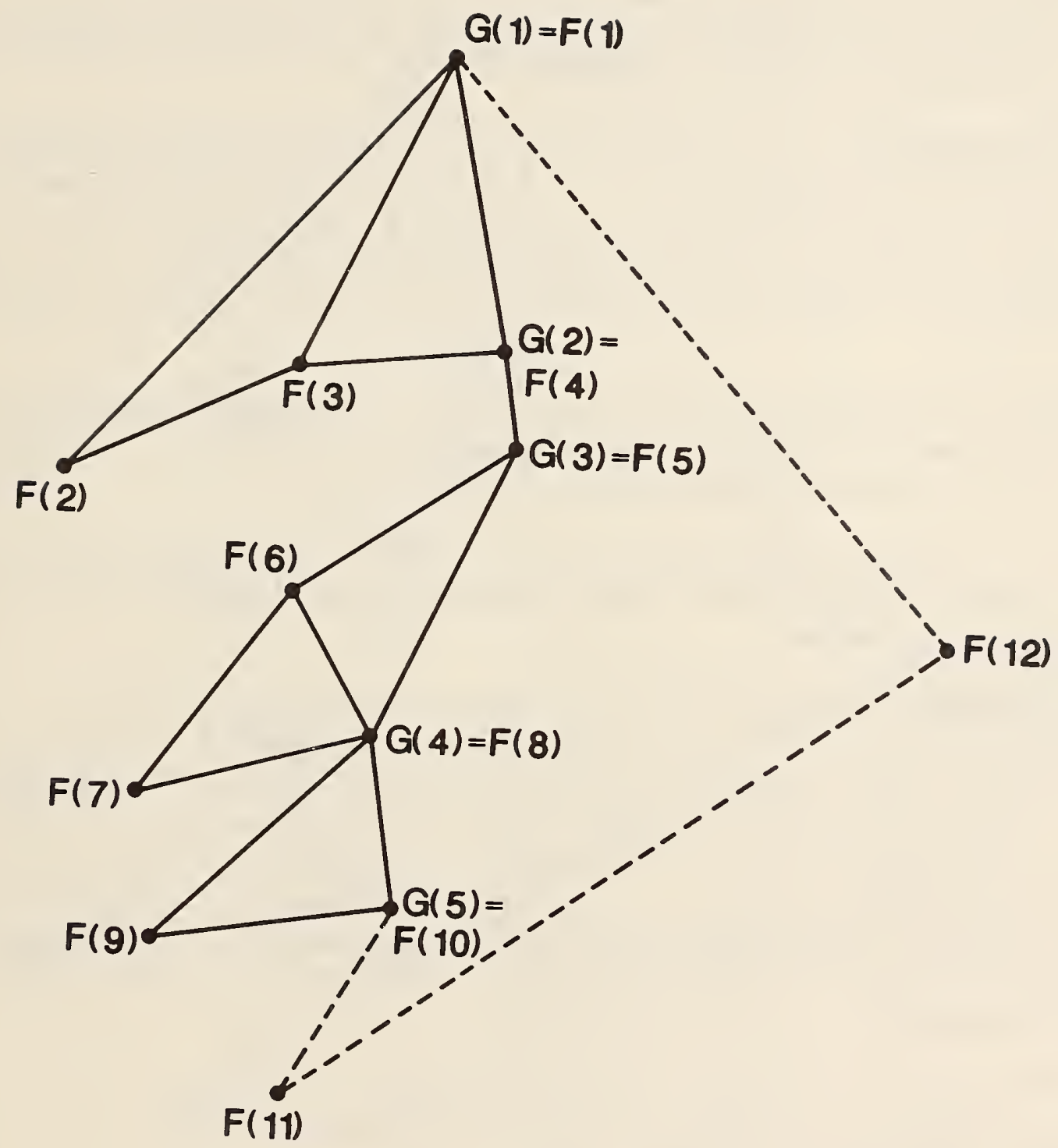

Figure 6: A polygon $R$, points $F(i), i=1, \ldots, r$, for $r$ equal to 12 , and the corresponding triangulation $T$ and points $G(j), j=1, \ldots, J$, at the start of the execution of line 6 of EDGSTR for $I$ and $J$ equal to 10 and 5 , respectively. 
8. Given $t$ in $T$, there exist integers $j, k, l, i_{1}, i_{2}, i_{3}, 1 \leq j \leq J-1$, $1 \leq k \leq i_{1}<i_{2}<i_{3} \leq l \leq I$, such that $G(j)$ and $G(j+1)$ equal $F(k)$ and $F(l)$, respectively, and $\triangle F\left(i_{1}\right) F\left(i_{2}\right) F\left(i_{3}\right)$ identifies $t$.

9. Given integers $j, k, l, 1 \leq j \leq J-1,1 \leq k<l \leq I$, such that $G(j)$ and $G(j+1)$ equal $F(k)$ and $F(l)$, respectively, if $k+1<l$ then (i) the union of the triangles in $T$ of the form $\Delta F\left(i_{1}\right) F\left(i_{2}\right) F\left(i_{3}\right), k \leq i_{1}<i_{2}<i_{3} \leq l$, is a simple polygon that is star-shaped relative to $[G(j), G(j+1)]$, and (ii) the closed half-plane determined by a clockwise rotation of $\pi$ radians of the ray $G(j) \vec{G}(j+1)$ around $G(j)$ contains $F(i)$ for each $i$, $k \leq i \leq l$.

10. Given an integer $k, 1 \leq k<I$, such that $G(J-1)$ equals $F(k)$, the closed half-plane determined by a clockwise rotation of $\pi$ radians of the ray $G(J-2) \vec{G}(J-1)$ around $G(J-2)$ contains $F(i)$ for each $i, k \leq i<I$.

11. $T$ is a Delaunay triangulation for a subset of $\{F(i), i=1, \ldots, I\}$ constrained by the boundary of the generalized multiply-connected polygonal region that the union of the triangles in $T$ defines.

Let $j_{r}$ be the initial value of $J$ the first time line 6 is executed for $I$ equal to $r$. From the first criterion above and lines 3, 4 and 8 of EDGSTR, at the start of the execution of line 6 for $I$ equal to $r$ and $J$ equal to $j_{r}$, we must have that $G(1), G(J-1)$, and $G(J)$ equal $P_{1}$, $P_{r-1}$, and $P_{r}$, respectively. This, together with the second criterion (for the same $I$ and $J$ ) and the ordering of $P_{i}, i=1, \ldots, r$, in $B N D(R)$, implies that $m(G(j) \vec{G}(J), G(j) \vec{G}(j-1))$ is less than $\pi$ for each $j, j=2, \ldots, J-1$. Figure 7 illustrates this situation for the same $R$ and $F$ of Figure 6 . Thus, from the remaining criteria (for the same $I$ and $J$ ), it can be seen from lines 6-10 that at the end of the execution of EDGSTR for $I$ equal to $r$, the following conditions are met:

1. The triangles in $T$ have pair-wise disjoint interiors.

2. Each triangle in $T$ is contained in $R$.

3. For each $i, i=1, \ldots, r-1$, there exists a triangle in $T$ with $\left[P_{i}, P_{i+1}\right]$ as an edge.

4. There exists a triangle in $T$ with $\left[P_{1}, P_{r}\right]$ as an edge.

5. Given an edge of a triangle in $T$, if it is not contained in $B N D(R)$ then it is an edge of two triangles in $T$. 


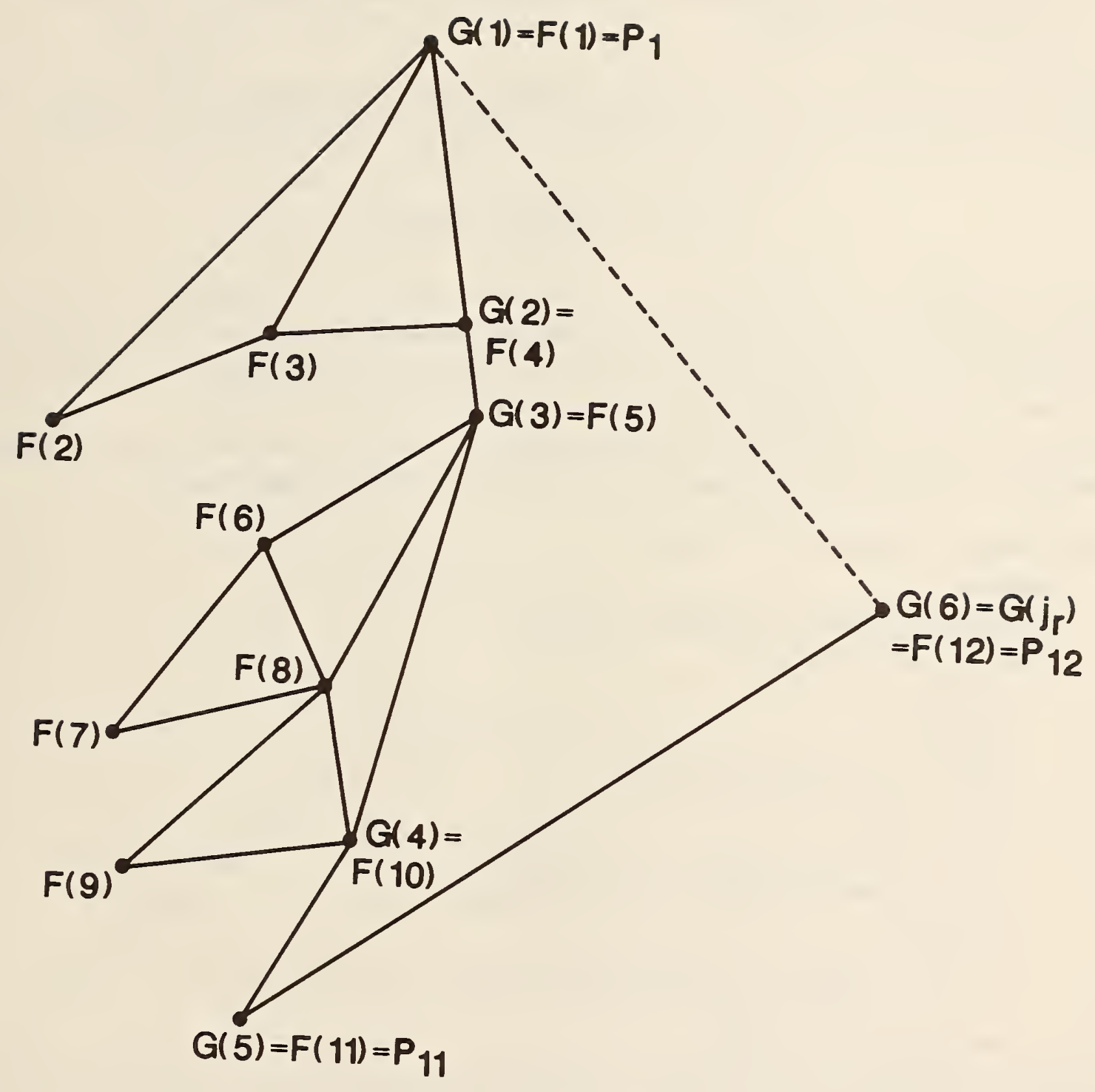

Figure 7: A polygon $R$, points $F(i), i=1, \ldots, r$, for $r$ equal to 12 , and the corresponding triangulation $T$ and points $G(j), j=1, \ldots, J$, at the start of the execution of line 6 of EDGSTR for $I$ and $J$ equal to $r$ and $j_{r}$, respectively. 
6. $T$ is a Delaunay triangulation for a subset of $S$ constrained by the boundary of the union of the triangles in $T$.

Since these conditions imply that the execution of EDGSTR produces a Delaunay triangulation $T$ for $S$ constrained by $B N D(R)$, the justification of EDGSTR is complete.

\section{The SEGMNT algorithm}

Let $S$ be a finite set of points in the plane. Given a positive integer $M$, let $e_{i}, i=1, \ldots, M$, be line segments in the plane that intersect only at points in $S$ and whose endpoints belong to $S$. Define a one-to-one function $D$ from $\{1, \ldots, M\}$ onto $E \equiv\left\{e_{i}, i=1, \ldots, M\right\}$ by setting $D(i)$ equal to $e_{i}$ for each $i, i=1, \ldots, M$.

In this section, we present and justify algorithm SEGMNT which computes a Delaunay triangulation $T^{*}$ for $S$ constrained by $E$. The outline of SEGMNT follows.

procedure SEGMNT $\left(T^{*}, S, D, M\right)$

\section{begin}

1. obtain a Delaunay triangulation $T^{*}$ for $S$;

2. for $I:=1$ until $M$ do

\section{begin}

3. $\quad T_{I}^{*}:=\emptyset$;

4. $\quad Q_{0}, Q_{1}:=$ endpoints of $D(I)$;

5. $\quad Q_{2}:=Q_{0}$;

6. $\quad H_{1}:=$ closed half-plane to 'left' of $Q_{0} \vec{Q}_{1}$;

7. $\quad H_{2}:=$ closed half-plane to 'left' of $Q_{1} \vec{Q}_{2}$;

8. $\quad$ flag $1:=1$;

9. if (each triangle in $T^{*}$ with $Q_{0}$ as a vertex is contained in $H_{1}$ ) then flag $1:=0$ else $J:=0$;

10. while $($ flag $1=1)$ do

$$
\text { begin }
$$

11. $J:=J+1$

12. $Q^{\prime \prime}, Q^{\prime \prime \prime}:=$ points in $S$ for which $\Delta Q_{J-1} Q^{\prime \prime} Q^{\prime \prime \prime}$

identifies a triangle in $T^{*}$ with $Q^{\prime \prime} \in H_{J}$ and $Q^{\prime \prime \prime} \notin H_{J}$;

13. $\quad$ if $\left(J=1\right.$ and $\left.Q^{\prime \prime} \in I N T\left(H_{J}\right)\right)$ then

$$
T_{I}^{*}:=T_{I}^{*} \cup\left\{\triangle Q_{J-1} Q^{\prime \prime} Q^{\prime \prime \prime}\right\} ;
$$

14.

$$
F_{J}(1):=Q_{J-1} ; F_{J}(2):=Q^{\prime \prime} \text {; }
$$


15.

$L:=2 ; Q^{\prime}:=Q^{\prime \prime \prime} ;$ flag $2:=1$;

16. while $($ flag $2=1)$ do

begin

17.

$Q^{\prime \prime \prime}:=$ point in $S$ for which $\Delta Q^{\prime} Q^{\prime \prime} Q^{\prime \prime \prime}$

identifies a triangle in $T^{*}$;

18.

if $\left(J=1\right.$ and $\left(Q^{\prime \prime} \in I N T\left(H_{J}\right)\right.$ or $\left.\left.Q^{\prime \prime \prime} \in I N T\left(H_{J}\right)\right)\right)$

then $T_{I}^{*}:=T_{I}^{*} \cup\left\{\Delta Q^{\prime} Q^{\prime \prime} Q^{\prime \prime \prime}\right\}$;

19. if $\left(Q^{\prime \prime \prime} \notin H_{J}\right)$ then $Q^{\prime}:=Q^{\prime \prime \prime}$ else

begin

20.

$L:=L+1 ; F_{J}(L):=Q^{\prime \prime \prime} ;$

21. if $\left(Q^{\prime \prime \prime} \neq Q_{J}\right)$ then $Q^{\prime \prime}:=Q^{\prime \prime \prime}$ else if $\left(J=1\right.$ and $\left.T_{I}^{*}=\emptyset\right)$ then flag1, flag $2:=0$

else

begin

22.

$r_{J}:=L$

23.

$\operatorname{EDGSTR}\left(T_{J}, F_{J}, r_{J}\right)$

24.

25. if $(J=1)$ then flag $2:=0$

else

begin

26.

$$
\begin{aligned}
& T^{*}:=\left(T^{*} \backslash T_{I}^{*}\right) \cup T_{1} \cup T_{2} ; \\
& \text { flag } 1, \text { flag } 2:=0
\end{aligned}
$$

end

end

end

end

end

end

end

Justification of SEGMNT. We show that the execution of SEGMNT produces a collection of triangles $T^{*}$ that is a Delaunay triangulation for $S$ constrained by $E$. Here, given a subset $E^{\prime}$ of $E$, a triangulation $T$ for $S$ constrained by $E^{\prime}$, and triangles $t$ and $t^{\prime}$ in $T$, we say that $t^{\prime}$ is adjacent to $t$ in $T$ relative to $E^{\prime}$ if the intersection of $t$ and $t^{\prime}$ is a line segment that is not contained in any of the line segments in $E^{\prime}$. In addition, we denote by $A D J\left(t, E^{\prime}\right)$ the set of points in $S$ that are vertices of triangles in $T$ adjacent to $t$ in $T$ relative to $E^{\prime}$, and say that $t$ satisfies the circle criterion in $T$ relative to $E^{\prime}$ if there does not exist a point of 
$A D J\left(t, E^{\prime}\right)$ inside the circumcircle of $t$. As in [5], we note that using arguments similar to those developed in [6,8], it can be shown that if each triangle in $T$ satisfies the circle criterion in $T$ relative to $E^{\prime}$ then $T$ is a Delaunay triangulation for $S$ constrained by $E^{\prime}$.

Let $I, J, T^{*}, T_{I}^{*}, H_{J}, T_{J}, F_{J}, r_{J}$ be as defined in the outline of SEGMNT. We show that at the end of each execution of line 25 of SEGMNT, $T^{*}$ is a Delaunay triangulation for $S$ constrained by $\left\{e_{i}: 1 \leq i \leq I\right\}$. Clearly, for $I$ equal to $M$ this is the desired conclusion.

From line 1 , at the start of the execution of line 3 for $I$ equal to $1, T^{*}$ is a Delaunay triangulation for $S$ constrained by $\left\{e_{i}: 1 \leq i \leq I-1\right\}$. Thus, we assume by induction on $I$ that given $k, 1 \leq k \leq M$, at the start of the execution of line 3 for $I$ equal to $k, T^{*}$ is a Delaunay triangulation for $S$ constrained by $\left\{e_{i}: 1 \leq i \leq I-1\right\}$, and show that at the end of the execution of line 25 for $I$ equal to $k, T^{*}$ is a Delaunay triangulation for $S$ constrained by $\left\{e_{i}: 1 \leq i \leq I\right\}$.

For each $J, J=1,2$, at the end of each execution of line 22 , let $R_{J}$ be defined as the intersection of $H_{J}$ with the union of the triangles in $T_{I}^{*}$. From the definitions of $T_{I}^{*}, H_{J}$, $F_{J}$, and $r_{J}$, for each $J, J=1,2$, if $R_{J}$ is not empty for $I$ equal to $k$, we may assume without any loss of generality that $R_{J}$ is a simple polygon that is star-shaped relative to $\left[F_{J}(1), F_{J}\left(r_{J}\right)\right]=D(I),\left\{F_{J}(L), L=1, \ldots, r_{J}\right\}$ equals $S \cap R_{J}$ and contains $\operatorname{VTX}\left(R_{J}\right)$, the points $F_{J}(L), L=1, \ldots, r_{J}$, appear in $B N D\left(R_{J}\right)$ in a counterclockwise direction around $I N T\left(R_{J}\right)$, and $D(I)$ intersects $S$ exactly at its vertices. Thus, for $I$ equal to $k$ and for each $J, J=1,2$, if $R_{J}$ is not empty EDGSTR can be executed in line 23 for $F_{J}$ and $r_{J}$ to produce a Delaunay triangulation $T_{J}$ for $S \cap R_{J}$ constrained by $B N D\left(R_{J}\right)$, and at the end of the execution of line 25 for the same $I, T^{*}$ is a triangulation for $S$. In addition, since $D(I)$ does not intersect the interior of each triangle in $T^{*}$, it follows from the induction hypothesis and the definitions of $E, T_{1}$, and $T_{2}$, that $T^{*}$ is a triangulation for $S$ constrained by $\left\{e_{i}: 1 \leq i \leq I\right\}$. Finally, let $t$ be a triangle in $T^{*}$ at the end of the execution of line 25 for $I$ equal to $k$, and assume $R_{J}$ for the same $I$ is not empty for each $J, J=1,2$. If $t$ belongs to $T^{*} \backslash\left(T_{1} \cup T_{2}\right)$ then from the induction hypothesis it follows that $t$ satisfies the circle criterion in $T^{*}$ relative to $\left\{e_{i}: 1 \leq i \leq I\right\}$. Else, if $t$ belongs to $T_{1}$ then all triangles adjacent to $t$ in $T^{*}$ relative to $\left\{e_{i}: 1 \leq i \leq I\right\}$ must belong to $T^{*} \backslash T_{2}$, and since $T_{1}$ is a Delaunay triangulation for $S \cap R_{1}$ constrained by $B N D\left(R_{1}\right)$, we must have that $t$ satisfies the circle criterion in $T^{*}$ relative to $\left\{e_{i}: 1 \leq i \leq I\right\}$. Similarly, if $t$ belongs to $T_{2}$ the same conclusion is obtained. Thus, by induction on $I$, we have shown that at the end of each execution of line $25, T^{*}$ is a Delaunay triangulation for $S$ constrained by $\left\{e_{i}: 1 \leq i \leq I\right\}$, and the justification of SEGMNT is complete. 


\section{Proof of complexity}

In this section, we assume that $S, E, M, e_{i}, i=1, \ldots, M$, are as defined in the previous section, and that the points in $S$ have been chosen independently from a uniform distribution on a square in the plane. Letting $N$ represent the cardinality of $S$, we then prove that for fixed $M$ the expected time required by SEGMNT to produce a Delaunay triangulation for $S$ constrained by $E$ is $O(N)$.

First, we introduce additional terminology. Let $B$ denote the square from which $S$ has been chosen, and let $d$ denote the Euclidean length of each of its sides. Let $K$ be defined as the floor of $N^{1 / 2}$, i. e. the largest integer less than or equal to $N^{1 / 2}$, and assume that $B$ has been divided into $K^{2}$ equal-sized square cells. Let $L(N)$ be defined as the floor of $\log N$ where $\log$ denotes the natural $\operatorname{logarithm}$, and let $B^{\prime}$ denote the square obtained by surrounding $B$ with $L(N)+1$ layers of cells of the type into which $B$ has been divided. Let $S^{\prime}$ denote the set of points that are centroids of cells in the $L(N)+1$ new layers. Given $e$ in $E$, let $|e|$ denote the Euclidean length of $e$. Given a finite set $X$ of points in the plane and points $P$ and $P^{\prime}$ in $X, P \neq P^{\prime}$, call $P^{\prime}$ a Delaunay neighbor of $P$ relative to $X$ if in some Delaunay triangulation for $X$ there exists a triangle with $P$ and $P^{\prime}$ as vertices. Given $G$, a finite nonempty set in the plane, and $G_{1}, G_{2}$, nonempty subsets of $G$, let $V\left(G, G_{1}, G_{2}\right)$ denote the set of points in $G_{2}$ that are Delaunay neighbors of points in $G_{1}$ relative to $G$. Given $e$ in $E$, let $V(e)$ represent the union of the sets $V(S \cup\{P\},\{P\}, S)$ for $P$ in $e$, let $V^{\prime}(e)$ represent the union of the sets $V\left(S \cup S^{\prime} \cup\{P\},\{P\}, S\right)$ for $P$ in $e$, and let $\overline{S(e)}$ represent the union of the sets $V\left(S \cup S^{\prime} \cup\{P\right.$. $\left.\}, S^{\prime}, S\right)$ for $P$ in $e$. Finally, let $\bar{S}$ represent the set $V\left(S \cup S^{\prime}, S^{\prime}, S\right)$.

Next, based on the terminology just defined, we list and prove some observations, the last of which establishes our assertion about the complexity of SEGMNT. Here, $I, J, F_{J}$, and $r_{J}$ are as defined in the outline of SEGMNT.

1. For each $e$ in $E, \overline{S(e)} \subseteq \bar{S}$.

2. For each $e$ in $E, V(e) \subseteq V^{\prime}(e) \cup \overline{S(e)} \subseteq V^{\prime}(e) \cup \bar{S}$.

3. For each $e$ in $E, S \cap e \subseteq V(e)$.

4. Given points $Q_{1}$ and $Q_{2}$ in $S$, if $Q_{1}$ is a Delaunay neighbor of $Q_{2}$ relative to $S$ then $Q_{1}$ and $Q_{2}$ belong to $V(S \cup\{P\},\{P\}, S)$ for each point $P$ in $\left(Q_{1}, Q_{2}\right)$.

5. Given an integer $i, 1 \leq i \leq M$, for $I$ equal to $i$ the points $F_{J}(L), L=1, \ldots, r_{J}$, $J=1,2$, are contained in $\cup_{l=1}^{i} V\left(e_{l}\right) \subseteq\left(\cup_{l=1}^{i} V^{\prime}\left(e_{l}\right)\right) \cup \bar{S}$. 
6. For each $e$ in $E$, the expected number of points in $V^{\prime}(e)$ is bounded above by $(|e| / d)$. $O\left(N^{1 / 2}\right)$.

7. The expected number of points in $\bar{S}$ is $O\left(N^{1 / 2}\right)$.

8. For fixed $M$, the expected time required by SEGMNT to produce a Delaunay triangulation for $S$ constrained by $E$ is bounded above by

$$
O(N)+\sum_{i=1}^{M}\left(\left(\left(\sum_{l=1}^{i}\left|e_{l}\right| / d\right)+1\right)^{2} \cdot O(N)\right)=\left(1+O\left(M^{3}\right)\right) \cdot O(N)=O(N) .
$$

Proof of first observation. Let $e$ be a line segment in $E$, and let $Q$ be a point in $\overline{S(e)}$. By the definition of $\overline{S(e)}, Q$ is in $S$ and for some point $P$ in $e$ there exists a point $Q^{\prime}$ in $S^{\prime}$ such that $Q$ is a Delaunay neighbor of $Q^{\prime}$ relative to $S \cup S^{\prime} \cup\{P\}$. Thus, we must have that either (i) $Q$ and $Q^{\prime}$ are adjacent points on the convex hull of $S \cup S^{\prime} \cup\{P\}$ or (ii) the line segment $\left[Q, Q^{\prime}\right]$ is an edge of two triangles in some Delaunay triangulation $T^{\prime}$ for $S \cup S^{\prime} \cup\{P\}$. If (i) occurs then $Q$ and $Q^{\prime}$ are also adjacent points on the convex hull of $S \cup S^{\prime}$ so that $Q$ is a Delaunay neighbor of $Q^{\prime}$ relative to $S \cup S^{\prime}$ and, therefore, $Q$ is a point in $\bar{S}$. Else, if (ii) occurs then for some point $Q^{\prime \prime}$ in $S \cup S^{\prime}$ there exists a triangle $t$ in $T^{\prime}$ such that $Q, Q^{\prime}$, and $Q^{\prime \prime}$ are vertices of $t$. By the definition of $T^{\prime}$ there does not exist a point of $S \cup S^{\prime}$ inside the circumcircle of $t$. Thus, $t$ is a triangle in some Delaunay triangulation for $S \cup S^{\prime}$ and, therefore, $Q$ belongs to $\bar{S}$.

Proof of second observation. Through slight modifications of a proof in [2], it can be shown that if $X$ and $Y$ are finite nonempty sets in the plane and $X^{\prime}$ is a nonempty subset of $X$ then

$$
V\left(X, X^{\prime}, X\right) \subseteq V\left(X \cup Y, X^{\prime}, X\right) \cup V(X \cup Y, Y, X) .
$$

Thus, given $e$ in $E$ and $P$ in $e$, we must have that

$$
\begin{gathered}
V(S \cup\{P\},\{P\}, S)=V(S \cup\{P\},\{P\}, S \cup\{P\}) \\
\subseteq V\left(S \cup S^{\prime} \cup\{P\},\{P\}, S \cup\{P\}\right) \cup V\left(S \cup S^{\prime} \cup\{P\}, S^{\prime}, S \cup\{P\}\right) \\
\subseteq V\left(S \cup S^{\prime} \cup\{P\},\{P\}, S\right) \cup V\left(S \cup S^{\prime} \cup\{P\}, S^{\prime}, S\right) \cup\{P\},
\end{gathered}
$$

and since $P$ does not belong to $V(S \cup\{P\},\{P\}, S)$ it follows that

$$
V(S \cup\{P\},\{P\}, S) \subseteq V\left(S \cup S^{\prime} \cup\{P\},\{P\}, S\right) \cup V\left(S \cup S^{\prime} \cup\{P\}, S^{\prime}, S\right),
$$


which together with the first observation implies the desired result.

Proof of third observation. Let $e$ be a line segment in $E$, and let $P^{\prime}$ be a point in $S \cap e$. The assertion follows, since for $P$ in $e$ sufficiently close to $P^{\prime}, P^{\prime}$ is in $V(S \cup\{P\},\{P\}, S)$.

Proof of fourth observation. Let $Q_{1}$ and $Q_{2}$ be points in $S$, and assume that $Q_{1}$ is a Delaunay neighbor of $Q_{2}$ relative to $S$. Let $P$ be a point in $\left(Q_{1}, Q_{2}\right)$, let $T^{\prime}$ be a Delaunay triangulation for $S \cup\{P\}$, and let $T^{\prime}\left(Q_{1}\right)$ be the collection of triangles in $T^{\prime}$ with $Q_{1}$ as a vertex. If $Q_{1}$ does not belong to $V(S \cup\{P\},\{P\}, S)$ then $P$ is neither inside nor on the circumcircle of each triangle in $T^{\prime}\left(Q_{1}\right)$. Thus, (i) the points that are vertices of triangles in $T^{\prime}\left(Q_{1}\right)$ belong to $S$, and (ii) $Q_{2}$ is neither inside nor on the circumcircle of each triangle in $T^{\prime}\left(Q_{1}\right)$. But this is a contradiction, since from (i) and the definition of $T^{\prime}$ a Delaunay triangulation for $S$ exists in which the collection of triangles with $Q_{1}$ as a vertex equals $T^{\prime}\left(Q_{1}\right)$ so that from (ii) $Q_{1}$ can not be a Delaunay neighbor of $Q_{2}$ relative to $S$. Thus, $Q_{1}$ belongs to $V(S \cup\{P\},\{P\}, S)$, and similarly, so does $Q_{2}$.

Proof of fifth observation. Let $T^{*}$ be as defined in the outline of SEGMNT. Given an integer $i, 1 \leq i \leq M$, let $T^{* i}$ equal $T^{*}$ at the start of the execution of line 3 of SEGMNT for $I$ equal to $i$, and let $S^{i}$ equal the set $\left\{F_{J}(L), L=1, \ldots, r_{J}, J=1,2\right\}$ for $I$ equal to $i$. In what follows, we show by induction on $i$ that $S^{i} \subseteq \cup_{l=1}^{i} V\left(e_{l}\right)$ for each $i, 1 \leq i \leq M$. The rest of the observation will follow from the second observation above.

Given $i, 1 \leq i \leq M$, let $Q$ be a point in $S^{i}$. It follows that either (1) $Q$ is a point in $S \cap e_{i}$, or (2) for some point $Q^{\prime}$ in $S$ and some triangle $t$ in $T^{* i}, Q$ and $Q^{\prime}$ are distinct vertices of $t$, and $\left(Q, Q^{\prime}\right)$ intersects the relative interior of $e_{i}$. If (1) occurs then by the third observation above $Q$ is in $V\left(e_{i}\right)$. Else, if (2) occurs, we assume inductively that if $P_{1}$ and $P_{2}$ are distinct vertices of a triangle in $T^{* i}$ then either (a) $i \geq 1$ and $P_{1}$ is a Delaunay neighbor of $P_{2}$ relative to $S$, or (b) $i>1$, and $P_{1}$ and $P_{2}$ belong to $\cup_{l=1}^{i-1} V\left(e_{l}\right)$. It then follows from (a), (b), and the fourth observation above that $Q$ is in $\cup_{l=1}^{i} V\left(e_{l}\right)$, and from the definition of $T^{* i}$, for $i<M$ the induction assumption holds when $i$ is replaced by $i+1$.

Proof of sixth observation. As in [1], given a point $P$ in the plane, a finite subset $X$ of the plane, and a collection $O$ of octants around $P$ as shown in Figure 8, we say that $P$ is closed in $X$ relative to $O$ if at least one point in $X$ different from $P$ is contained in each of the octants in $O$.

Let $e$ be a line segment in $E$, let $K(e)$ represent the floor of $(|e| / d) \cdot N^{1 / 2}$, and without regard to any specific order, let $Q_{0}$ and $Q_{K(e)}$ denote the endpoints of $e$. For each $k, k=0, \ldots, K(e)$, let $P_{k}$ denote the point

$$
Q_{0}+(k / K(e)) \cdot\left(Q_{K(e)}-Q_{0}\right)
$$

and let $O_{k}$ denote the collection of octants around $P_{k}$ for which the interior of each octant 


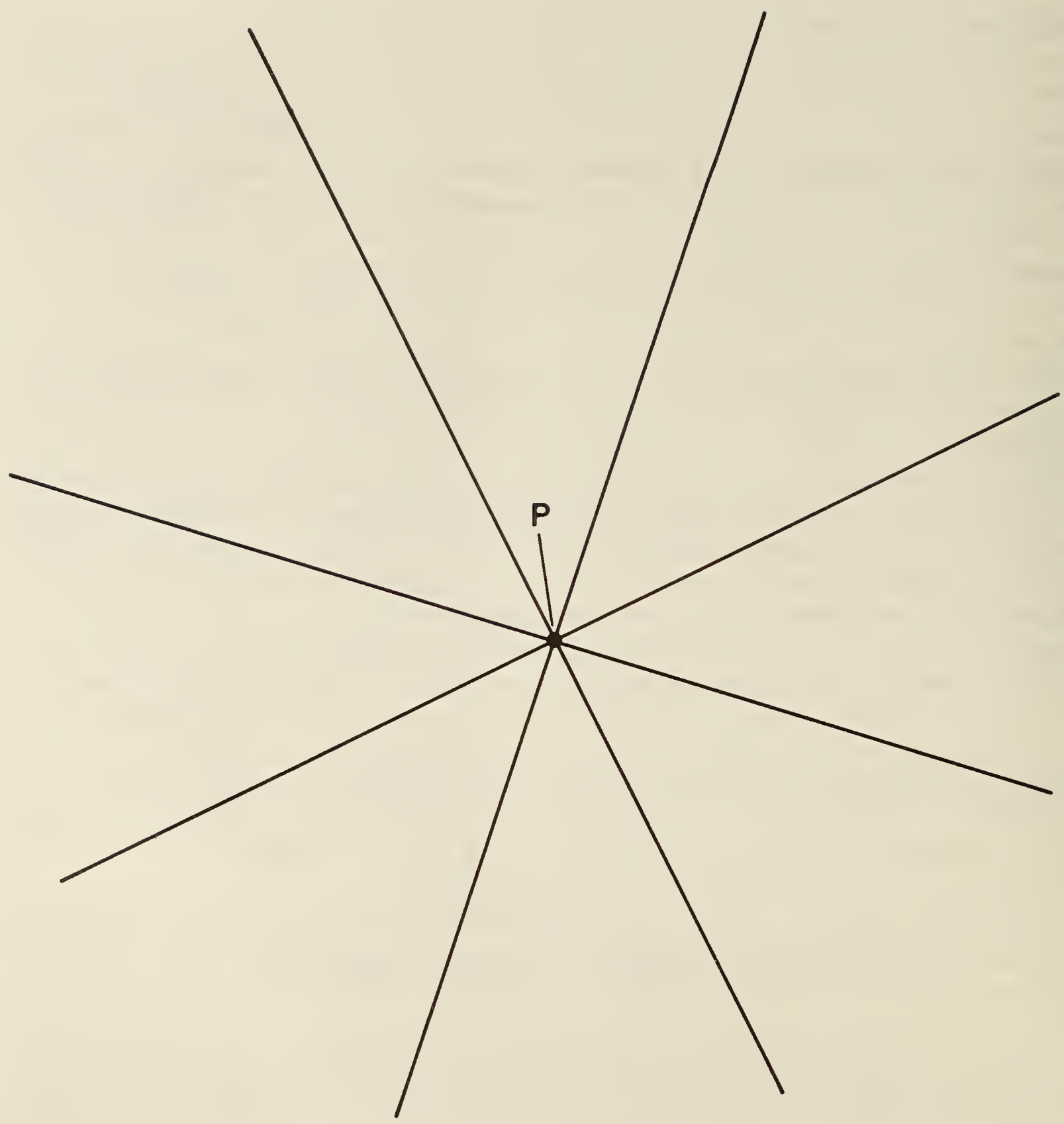

Figure 8: A point $P$ in the plane and a collection of octants around it. 
does not intersect $e$. Figure 9 illustrates an example of $P_{k}, O_{k}, k=1, \ldots, K(e)$.

Let $p_{1}$ be the probability that for each $k, k=0, \ldots, K(e), P_{k}$ is closed in $S \cup S^{\prime}$ relative to $O_{k}$, and let $t_{1}$ be the expected number of points in $V^{\prime}(e)$ when for each $k, k=0, \ldots, K(e)$, $P_{k}$ is closed in $S \cup S^{\prime}$ relative to $O_{k}$. Let $p_{2}$ and $t_{2}$ be similarly defined, respectively, for the event in which for some $k, 0 \leq k \leq K(e), P_{k}$ is not closed in $S \cup S^{\prime}$ relative to $O_{k}$.

Given $k, 0 \leq k \leq K(e), P_{k}$ is not in any of the outermost $L(N)$ layers of cells of $B^{\prime}$. Thus, it follows from the selection of $S$ and $S^{\prime}$, and results in [1], that if $P_{k}$ is closed in $S \cup S^{\prime}$ relative to $O_{k}$ then the expected number of points in $V\left(S \cup S^{\prime} \cup\left\{P_{k}\right\},\left\{P_{k}\right\}, S \cup S^{\prime}\right)$ is $O(1)$. In addition, we note that for each $k, k=1, \ldots, K(e)$, the length of $\left[P_{k-1}, P_{k}\right]$ equals $|e| / K(e) \approx d / N^{1 / 2}$ (the length of each side of a cell), and $e$ equals $\cup_{k=1}^{K(e)}\left[P_{k-1}, P_{k}\right]$. Hence, it follows from the geometry of the octants around the points $P_{k}, k=0, \ldots, K(e)$, as illustrated in Figure 9, that if for each $k, k=0, \ldots, K(e), P_{k}$ is closed in $S \cup S^{\prime}$ relative to $O_{k}$, then the expected number of points in $\cup_{P \epsilon e} V\left(S \cup S^{\prime} \cup\{P\},\{P\}, S \cup S^{\prime}\right)$ is $K(e) \cdot O(1)$. Therefore,

$$
t_{1}=K(e) \cdot O(1)=(|e| / d) \cdot O\left(N^{1 / 2}\right) .
$$

On the other hand, if for some $k, 0 \leq k \leq K(e), P_{k}$ is not closed in $S \cup S^{\prime}$ relative to $O_{k}$, then using an argument from [1], the probability of this occurring is bounded above by $8 \cdot(K(e)+1) \cdot e^{-O\left(L(N)^{2}\right)}$. Hence,

$$
p_{2} \leq 8 \cdot(K(e)+1) \cdot e^{-O\left(L(N)^{2}\right)} .
$$

Finally, $t_{2}$ is clearly bounded above by the number of points in $S$. Therefore,

$$
t_{2} \leq N .
$$

Thus, if $t$ is the expected number of points in $V^{\prime}(e)$, then

$$
\begin{aligned}
t & =p_{1} \cdot t_{1}+p_{2} \cdot t_{2} \\
& =1 \cdot(|e| / d) \cdot O\left(N^{1 / 2}\right)+8 \cdot(K(e)+1) \cdot e^{-O\left(L(N)^{2}\right)} \cdot N \\
& =(|e| / d) \cdot O\left(N^{1 / 2}\right)+8 \cdot(K(e)+1) \cdot O(1) \\
& =(|e| / d) \cdot O\left(N^{1 / 2}\right)+(|e| / d) \cdot O\left(N^{1 / 2}\right) \\
& =(|e| / d) \cdot O\left(N^{1 / 2}\right) .
\end{aligned}
$$

Proof of seventh observation. Let $S^{\prime \prime}$ represent the set of points in $S^{\prime}$ contained in the first layer of cells in $B^{\prime}$ that surrounds $B$. From the definition of $S^{\prime}$, points in $S^{\prime \prime}$ are the only points in $S^{\prime}$ that can be Delaunay neighbors of points in $S$ relative to $S \cup S^{\prime}$. Since points 


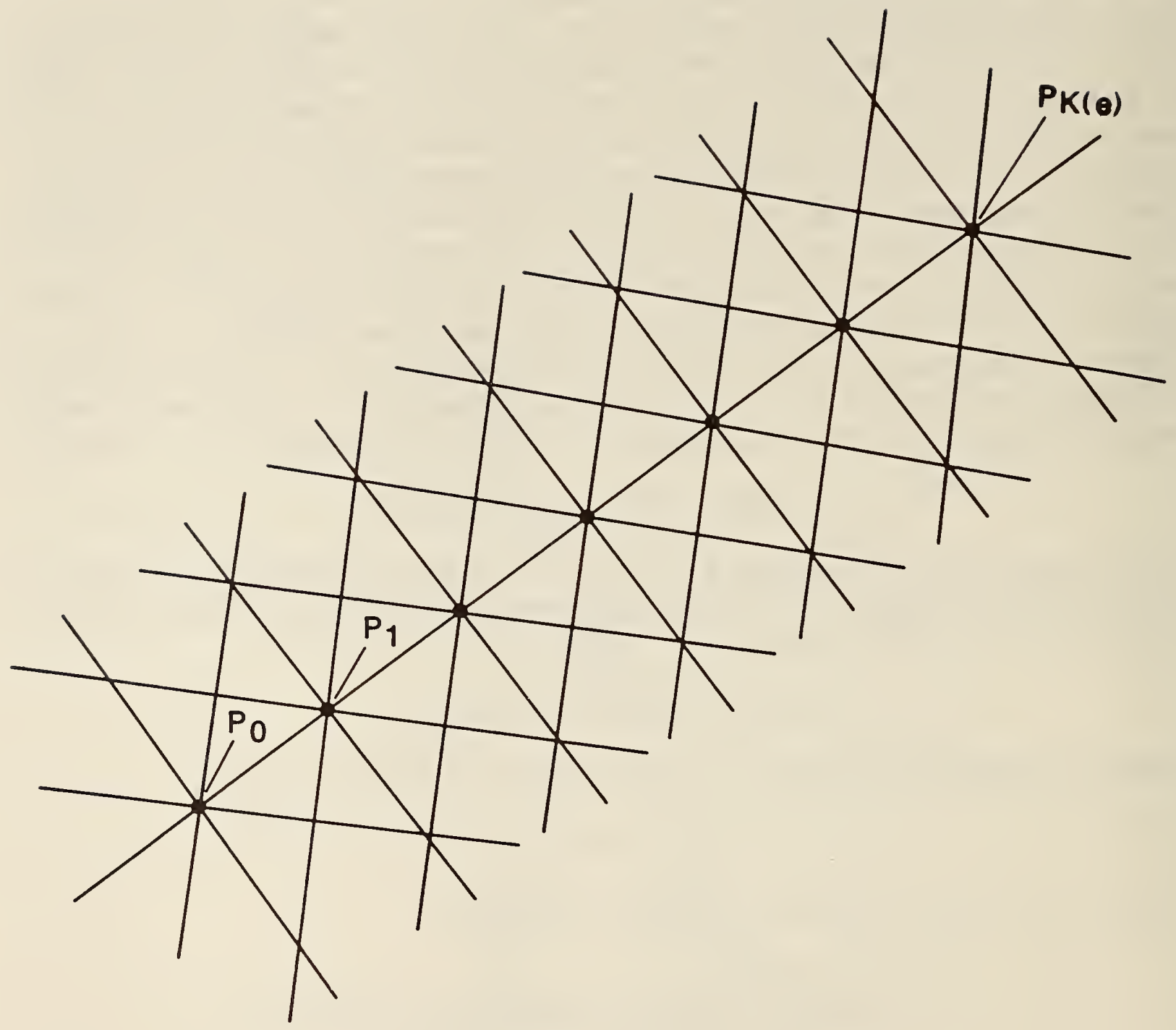

Figure 9: The points and collections of octants $P_{k}, O_{k}, k=0, \ldots, K(e)$. 
in $S^{\prime \prime}$ are not contained in any of the outermost $L(N)$ layers of cells of $B^{\prime}$, it follows from results in [1] that the expected number of points in $S \cup S^{\prime}$ that are Delaunay neighbors of a given point in $S^{\prime \prime}$ relative to $S \cup S^{\prime}$ is $O(1)$. Therefore, since the number of points in $S^{\prime \prime}$ is $O\left(N^{1 / 2}\right)$, we must have that the expected number of points in

$$
\bar{S}=V\left(S \cup S^{\prime}, S^{\prime}, S\right)=V\left(S \cup S^{\prime}, S^{\prime \prime}, S\right) \subseteq V\left(S \cup S^{\prime}, S^{\prime \prime}, S \cup S^{\prime}\right)
$$

is $O\left(N^{1 / 2}\right) \cdot O(1)=O\left(N^{1 / 2}\right)$.

Proof of eighth observation. Let $T_{J}$ be as defined in the outline of SEGMNT. Given $i$, $1 \leq i \leq M$, it follows from the fifth observation above that for $I$ equal to $i$ the points $F_{J}(L)$, $L=1, \ldots, r_{J}, J=1,2$, are contained in $\cup_{l=1}^{i} V\left(e_{l}\right) \subseteq\left(\cup_{l=1}^{i} V^{\prime}\left(e_{l}\right)\right) \cup \bar{S}$. In addition, from the outline of EDGSTR, we obtain that for $I$ equal to $i$ the worst-case time required by the execution of EDGSTR to produce $T_{J}, J=1,2$, in line 23 of SEGMNT is $O\left(r_{J}^{2}\right)$. Therefore, from the sixth and seventh observations above, we must have that for $I$ equal to $i$ the expected time required by the execution of EDGSTR to produce $T_{J}, J=1,2$, is bounded above by

$$
\begin{aligned}
\left(\left(\sum_{l=1}^{i}\left(\left(\left|e_{l}\right| / d\right) \cdot O\left(N^{1 / 2}\right)\right)\right)+O\left(N^{1 / 2}\right)\right)^{2} & =\left(\left(\left(\sum_{l=1}^{i}\left|e_{l}\right| / d\right)+1\right) \cdot O\left(N^{1 / 2}\right)\right)^{2} \\
& =\left(\left(\sum_{l=1}^{i}\left|e_{l}\right| / d\right)+1\right)^{2} \cdot O(N) .
\end{aligned}
$$

The result now follows by assuming that the Delaunay triangulation for $S$ obtained in line 1 of SEGMNT is constructed by applying to $S$ an expected $O(N)$ algorithm $[1,2,9,10]$.

\section{Summary}

We have presented a simple algorithm for computing a Delaunay triangulation for a finite set $S$ of points in the plane constrained by a finite collection $E$ of line segments. The algorithm is based on the simple idea of constructing first a Delaunay triangulation for $S$, and then 'repairing' this triangulation as each line segment in $E$ is incorporated into the previously obtained triangulation. As it turns out, the 'repairing' of the triangulation is an easy process, since it involves computing the Delaunay triangulation of subsets of edge star-shaped simple polygons constrained by the boundaries of the polygons. Given that $N$ is the number of points in $S$, and $M$ is the number of line segments in $E$, we have also shown that if the points in $S$ are chosen independently from a uniform distribution on a square, then the 
expected time required by the algorithm to produce the desired triangulation is bounded above by $O\left(M^{3} \cdot N\right)$. Actually, depending on the lengths of the line segments and their locations with respect to the boundary of the square, we expect this time to be $O(M \cdot N)$ or better for $N$ large relative to $M$, since in this case most of the triangles that are eliminated during the 'repairing' of the triangulation belong to the Delaunay triangulation for $S$. At any rate, for fixed $M$, the expected time required by the algorithm to produce a Delaunay triangulation for $S$ constrained by $E$ is $O(N)$. Finally, we report that an implementation of the algorithm has just been completed at the National Bureau of Standards.

\section{Acknowledgments}

The author gratefully acknowledges Professor James F. Lawrence and Mr. Donald J. Orser for reading the manuscript and offering their criticism and helpful suggestions. He also wishes to thank Dr. Christoph Witzgall for his encouragement during the preparation of the manuscript. 


\section{References}

[1] J. L. Bentley, B. W. Weide and A. C. Yao, Optimal expected-time algorithms for closest point problems, ACM Trans. Math. Software 6, No. 4, 1980, 563-580.

[2] J. Bernal, Expected $O(N)$ and $O\left(N^{4 / 3}\right)$ algorithms for constructing Voronoi diagrams in two and three dimensions, NBSIR 87-3679, National Bureau of Standards, Washington, D. C., 1987.

[3] L. P. Chew, There is a planar graph almost as good as the complete graph, in Proc. $2^{\text {nd }}$ ann. Symp. on Computational Geometry, Yorktown Heights, 1986, 169-177.

[4] L. P. Chew, Constrained Delaunay triangulations, in Proc. $3^{\text {rd }}$ ann. Symp. on Computational Geometry, Waterloo, Ontario, 1987, 215-222.

[5] L. De Floriani, B. Falcidieno and C. Pienovi, Delaunay-based representation of surfaces defined over arbitrarily shaped domains, Comput. Vision Graphics Image Process. 32, 1985, 127-140.

[6] C. L. Lawson, Software for $C^{1}$ surface interpolation, Math. Software III, (J. R. Rice, Ed.), pp. 161-194, Academic Press, New York, 1977.

[7] D. T. Lee, Proximity and reachability in the plane, Tech. Rep. R-831, Coordinated Science Laboratory, University of Illinois, 1978.

[8] D. T. Lee and B. J. Schachter, Two algorithms for constructing a Delaunay triangulation, Int. J. Comput. Inform. Sci. 9, No. 3, 1980, 219-242.

[9] A. Maus, Delaunay triangulation and the convex hull of $N$ points in expected linear time, BIT 24, 1984, 151-163.

[10] T. Ohya, M. Iri and K. Murota, A fast Voronoi-diagram algorithm with quaternary tree bucketing, Inform. Proc. Letters 18, No. 4, 1984, 227-231.

[11] M. I. Shamos, Computational geometry, Ph.D. dissertation, Yale University, 1978.

[12] M. I. Shamos and D. Hoey, Closest-point problems, in Proc. $16^{\text {th }}$ Ann. IEEE Symp. on the Foundations of Comp. Sci., 1975, pp. 151-162. 

NBS.114A (REV. 2.80)

U.S. DEPT. OF COMM.

BIBLIOGRAPHIC DATA

SHEET (See in structions)

1. PUBLICATION OR

REPORT NO.

NIST/TN-1252

2. Performing Organ. Report No, 3. Publication Date

September 1988

4. TITLE AND SUBTITLE

On Constructing Delaunay Triangulations of Sets Constrained by Line Segments

5. AUTHOR(S)

Javier Bernal

6. PERFORMING ORGANIZATION (If joint or other than NBS, see instructions)

7. Contract/Grant No.

NATIONAL BUREAU OF STANDARDS

U.S. DEPARTMENT OF COMMERCE

GAITHERSBURG, MD 20899

8. Type of Report \& Period Covered

Fina 1

9. SPONSORING ORGANIZATION NAME AND COMPLETE ADDRESS (Stree, City, Stote, ZIP)

Same as item 6

10. SUPPLEMENTARY NOTES

Document describes a computer program; SF-185, FIPS Software Summary, is attached.

11. ABSTRACT (A 200-word or less factual summary of most significant information. If document includes a significant bibliography or literature survey. mention it here)

In this paper, we propose a simple algorithm for constructing a Delaunay triangulation for a finite set of points in the plane constrained by a finite collection of line segments. This algorithm constructs first a Delaunay triangulation for the set, and then generates from it a sequence of triangulations as each 1 ine segment is incorporated into the previously obtained triangulation. An expected time analysis shows the algorithm to be linear if the number of line segments is kept constant.

12. KEY WORDS (Six to twelve entries; alphabetical order; capitalize only proper names; and separate key words by semicolon s) algorithm; computational complexity; computational geometry; constrained Delaunay triangulation; expected time analysis; multiply-connected polygonal regions; Voronoi diagram

13. AVAILABILITY

[X] Unlimited

$\square$ For Official Distribution. Do Not Release to NTIS

[X] Order From Superintendent of Documents, U.S. Government Printing Office, Washington, D.C. 20402.

14. NO. OF PRINTED PAGES

33

[X] Order From National Technical Information Service (NTIS), Springfield, VA. 2216I

15. Price 


U.S. Department of Commerce

National Institute of Standards and Technology

(formerly National Bureau of Standards)

Gaithersburg, MD 20899

Official Business

Penalty for Private Use $\$ 300$

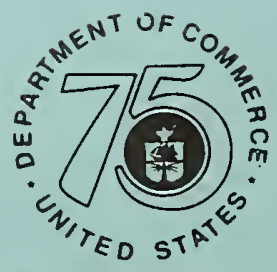

